TRANSACTIONS OF THE

AMERICAN MATHEMATICAL SOCIETY

Volume 352, Number 3, Pages 1345-1380

$\mathrm{S} 0002-9947(99) 02184-4$

Article electronically published on May 21, 1999

\title{
POSITIVE DEFINITE SPHERICAL FUNCTIONS ON OL'SHANSKII DOMAINS
}

\author{
JOACHIM HILGERT AND KARL-HERMANN NEEB
}

\begin{abstract}
Let $G$ be a simply connected complex Lie group with Lie algebra $\mathfrak{g}, \mathfrak{h}$ a real form of $\mathfrak{g}$, and $H$ the analytic subgroup of $G$ corresponding to $\mathfrak{h}$. The symmetric space $\mathcal{M}=H \backslash G$ together with a $G$-invariant partial order $\leq$ is referred to as an $\mathrm{Ol}^{\prime}$ shanskiŭ space. In a previous paper we constructed a family of integral spherical functions $\phi_{\mu}$ on the positive domain $\mathcal{M}^{+}:=\{H x: H x \geq$ $H\}$ of $\mathcal{M}$. In this paper we determine all of those spherical functions on $\mathcal{M}^{+}$ which are positive definite in a certain sense.
\end{abstract}

\section{INTRODUCTION}

Let $G$ be a simply connected complex Lie group with Lie algebra $\mathfrak{g}, \mathfrak{h}$ a real form of $\mathfrak{g}$, and $H$ the analytic subgroup of $G$ corresponding to $\mathfrak{h}$. Then $H \backslash G$ is a symmetric space which admits a $G$-invariant partial order $\leq$ associated to each closed convex $\operatorname{Ad}(H)$-invariant cone $C$ in $\mathfrak{h}$ which is generating but contains no lines. In fact, one can show that $H \exp (i C)$ is a closed subsemigroup of $G$ and the $G$-invariant order on $H \backslash G$ is given by

$$
H x \leq H y \quad \text { if } \quad y \in H \exp (i C) x .
$$

The space $\mathcal{M}=H \backslash G$ together with such an ordering is called an $\mathrm{Ol}^{\prime}$ shanskiu space (cf. [HÓ96]). The subset $\mathcal{M}^{+}:=\{H x: H x \geq H\}$ of $\mathcal{M}$ is called the positive domain of $\mathcal{M}$ and is a key example for what we will call an $\mathrm{Ol}^{\prime}$ shanskiu domain in this paper.

Generalizing results from [FHÓ94], we constructed in [HiNe96] a family of spherical functions $\varphi_{\mu}$ on $\mathcal{M}^{+}$via an integral formula similar to Harish-Chandra's integral representation of spherical functions on non-compact Riemannian symmetric spaces. These functions are parametrized by a subset $\mathfrak{t}^{*}+\mathcal{E}_{\mathcal{L}} \subseteq \mathfrak{t}_{\mathbb{C}}^{*}$, where $\mathfrak{t}$ is a suitable Cartan subalgebra of $\mathfrak{h}$. In this paper we introduce a concept of positive definite spherical functions on positive domains, and in the case described above we determine all of these. In particular we determine which of the integral spherical functions constructed in [HiNe96] are positive definite spherical functions (Theorem $5.2)$.

The natural framework for the study of spherical functions on positive domains of ordered symmetric spaces is that of symmetric involutive semigroups. In view

Received by the editors October 4, 1996 and, in revised form, July 1, 1997.

1991 Mathematics Subject Classification. Primary 22E46; Secondary 22A25.

Key words and phrases. Positive definite function, ordered symmetric space, holomorphic representation, spherical function, involutive semigroup. 
of future applications we use the general concepts in this paper, even though the main results are proved only in the case of $\mathrm{Ol}^{\prime}$ shanskil domains.

A symmetric involutive semigroup is a semigroup $S$ equipped with two commuting involutive selfmaps ${ }^{*}$ and $\tau$, one an antiautomorphism and one an automorphism. To each such semigroup one can associate a positive domain $P=$ $\left\{\tau(s)^{*} s: s \in S\right\}$. In the context of $\mathrm{Ol}^{\prime}$ shanskiur spaces we have (up to a covering which is used for technical reasons) $S=H \exp (i C)$ with $(h \exp (i X))^{*}=$ $\exp (i X) h^{-1}$ and $\tau=\mathrm{id}$, and hence the positive domain is the $\mathrm{Ol}^{\prime}$ shanskiu domain $P_{C}=\exp (i C) \cong \mathcal{M}^{+}$.

A function $\varphi: S \rightarrow \mathbb{C}$ is called positive definite if the formula $K(s, t)=\varphi\left(s t^{*}\right)$ defines a positive definite kernel on $S$. Associated to such a kernel is a reproducing kernel space $\mathcal{H}_{\varphi}$ of functions on $S$. If the right regular representation of $S$ yields a representation $\pi_{\varphi}: S \rightarrow B\left(\mathcal{H}_{\varphi}\right)$ of $S$ by bounded operators, we call $\varphi$ exponentially bounded.

Similarly, a function $\bar{\varphi}: P \rightarrow \mathbb{C}$ is called positive definite if the formula

$$
K(q(s), q(t))=\bar{\varphi}\left(q\left(s t^{*}\right)\right),
$$

where $q: S \rightarrow P$ is the canonical map defined by $q(s)=\tau(s)^{*} s$, defines a positive definite kernel on $P$. Here, $\bar{\varphi}$ is called exponentially bounded if the positive definite function $\bar{\varphi} \circ q$ on $S$ is exponentially bounded. The function $\bar{\varphi}$ is called spherical if the corresponding representation of $S$ on the associated reproducing kernel space $\mathcal{H}_{\bar{\varphi}}$ is irreducible.

The general approach to positive definite functions on semigroups and related objects is developed in [Ne99]. For the convenience of the reader we refer to that book for details also on background material like reproducing kernel spaces which could be found elsewhere (e.g. [Ar50]).

The first two sections of this paper are devoted to the basic definitions and examples related to symmetric involutive semigroups and positive definite spherical functions on the associated positive domains.

In Section 3 we provide an important technical tool in our classification of the positive definite spherical functions, the Lüscher-Mack correspondence, which connects the representation theory of the simply connected covering semigroup $S_{C}$ of $H \exp (i C)$ with the unitary representation theory of the simply connected $c$-dual group (cf. [HÓ96]) $G^{c}=\widetilde{H} \times \widetilde{H}$ of $G$. In fact, we present the Lüscher-Mack correspondence in greater generality than needed in this paper, since we expect it to play a similar if not more important role in the study of positive definite spherical functions for more general ordered symmetric spaces (cf. [FHÓ94], [KrNe96], and [KNÓ96].

Given the Lüscher-Mack correspondence, spherical functions on the positive domain $P_{C}$ of $S_{C}$ are closely related to $S_{C}$-spherical representations of $G^{c}$, and this relation is of crucial importance for the classification results alluded to above. Here an irreducible unitary representation $(\pi, \mathcal{H})$ of $G^{c}$ is called $S_{C^{-}}$-spherical if it is related to a representation $\rho$ of $S_{C}$ via the Lüscher-Mack correspondence and admits an $\widetilde{H}$-invariant linear functional on the dense subspace $\mathcal{H}^{0}:=\operatorname{span}\left(\rho\left(S_{C}\right) \cdot \mathcal{H}\right)$ of $\mathcal{H}$ satisfying certain continuity conditions. These representations are studied in Section 4.

Sections 5 and 6 contain the main results of this paper, notably the classification of the positive definite spherical functions on $\mathrm{Ol}^{\prime}$ shanskiu domains (Theorem 5.1) 
and the classification of the holomorphic positive definite spherical functions on $S_{C}$ (Theorem 6.2).

The last section contains a short discussion of possible generalizations. We have also included two appendices which contain some technical results related to the analytic extension of highest weight representations, considerably generalizing those of $\mathrm{Ol}^{\prime}$ shanskiı̌ in [Ols82].

\section{Symmetric involutive SEmigroups}

Let $S$ be a semigroup and ${ }^{*}: S \rightarrow S, s \mapsto s^{*}$ an involutive antiautomorphism of $S$. Then $\left(S,{ }^{*}\right)$ is called an involutive semigroup. We refer to [HiNe93, Chap. 9] and [Ne99, Chap. II] for the basic properties of involutive semigroups.

Definition 1.1. Let $\left(S,{ }^{*}\right)$ be an involutive semigroup and $\tau: S \rightarrow S$ be an involutive automorphism commuting with ${ }^{*}$. Then $\left(S,{ }^{*}, \tau\right)$ is called a symmetric involutive semigroup and the set $P:=\left\{\tau(s)^{*} s: s \in S\right\}$ is called the associated positive domain.

The semigroup $S$ acts on $P$ from the right by $x . s:=\tau(s)^{*} x s$, and the canonical map

$$
q: S \rightarrow P, \quad s \mapsto \tau(s)^{*} s
$$

is equivariant with respect to the right multiplication action of $S$ on itself and the action on $P$.

As far as the positive domain $P$ considered as an $S$-space is concerned, it seems at this point to be artificial to consider the two involutions $s \mapsto s^{*}$ and $\tau$ on $S$, because only the involutive antiautomorphism $s \mapsto \tau(s)^{*}$ shows up in the definition of $P$. The reason for keeping two involutions in the definition will become clear when we study invariance properties of kernels on $P$.

The class of examples which are of central importance in our context is positive domains related to symmetric spaces: Recall that a symmetric Lie group is a pair $(G, \tau)$, where $G$ is a Lie group and $\tau$ is an involutive automorphism of $G$. A symmetric Lie algebra $(\mathfrak{g}, \tau)$ is defined analogously. Note that if $(G, \tau)$ is a symmetric Lie group, then $(\mathfrak{g}, d \tau(e))$ is a symmetric Lie algebra. In such a situation we will simply write $\tau$ for $d \tau(e)$. We denote the group of $\tau$-fixed points in $G$ by $G^{\tau}$. If $H \subseteq G^{\tau}$ is an open subgroup, then $H \backslash G$ is called a symmetric space associated to the symmetric Lie algebra $(\mathfrak{g}, \tau)$.

If $(\mathfrak{g}, \tau)$ is a symmetric Lie algebra, then we write

$$
\mathfrak{h}=\mathfrak{g}^{\tau}=\{X \in \mathfrak{g}: \tau(X)=X\} \quad \text { and } \quad \mathfrak{q}=\{X \in \mathfrak{g}: \tau(X)=-X\}
$$

and note that $\mathfrak{g}=\mathfrak{h}+\mathfrak{q}$ is a direct vector space decomposition.

Example 1.2. Let $(G, \tau)$ be a simply connected symmetric Lie group and $H=G^{\tau}$ the fixed point group for $\tau$, which, in view of the simple connectedness of $G$, is connected ([Lo69, p.171]). Further let $C \subseteq \mathfrak{q}$ be an $\operatorname{Ad}(H)$-invariant generating closed convex cone which is regular, i.e., $\operatorname{Spec}(\operatorname{ad} X) \subseteq \mathbb{R}$ holds for all $X \in C$. Then, according to Lawson's Theorem ([HiNe93, Thm. 7.34, Cor. 7.35]), the set

$$
\Gamma(C):=H \exp (C)
$$

is a closed subsemigroup of $G$ and the mapping

$$
H \times C \rightarrow \Gamma(C), \quad(h, X) \mapsto h \exp (X)
$$

is a homeomorphism. 
Let $\widetilde{\Gamma}(C)$ denote the universal covering semigroup of $\Gamma(C)$ and note that we then have a homeomorphism

$$
\widetilde{H} \times C \rightarrow \widetilde{\Gamma}(C), \quad(h, X) \mapsto h \operatorname{Exp}(X),
$$

where $\operatorname{Exp}: C \rightarrow \widetilde{\Gamma}(C)$ is the lift of the exponential function with $\operatorname{Exp}(0)=e$.

We also write $\widetilde{\Gamma}\left(C^{0}\right)$ for the subsemigroup $\widetilde{H} \operatorname{Exp}\left(C^{0}\right)$ of $\widetilde{\Gamma}(C)$, where $C^{0}=\operatorname{int} C$ denotes the interior of the cone $C$. We have the involution $s=h \operatorname{Exp}(X) \mapsto s^{*}:=$ $\operatorname{Exp}(X) h^{-1}$ on $\widetilde{\Gamma}(C)$, defining on $\widetilde{\Gamma}(C)$ the structure of an involutive semigroup. Then

$$
P_{C}:=\left\{s^{*} s: s \in \widetilde{\Gamma}\left(C^{0}\right)\right\}=\operatorname{Exp}\left(C^{0}\right)
$$

is the positive domain associated to $\left(\widetilde{\Gamma}\left(C^{0}\right),{ }^{*}, \operatorname{id}_{\widetilde{\Gamma}(C)}\right)$, and $q: \widetilde{\Gamma}\left(C^{0}\right) \rightarrow P_{C}, s \mapsto s^{*} s$ is the corresponding canonical map.

Definition 1.3. If, in the situation of Example 1.2, $H$ is a real form of $G$ and $\tau$ the complex conjugation, we call the positive domain $P_{C}$ an $O l^{\prime}$ shanskiu domain.

The following construction will be useful in the study of holomorphic spherical functions (cf. §6).

Example 1.4. (a) Let $\left(S,{ }^{*}\right)$ be any involutive semigroup. The involution

$$
\left(s_{1}, s_{2}\right)^{*}:=\left(s_{1}^{*}, s_{2}^{*}\right)
$$

turns the double $S^{d}:=S \times S$ of $S$ into an involutive semigroup. In addition, we consider the involutive automorphism $\tau\left(s_{1}, s_{2}\right)=\left(s_{2}, s_{1}\right)$, which commutes with * Then $\left(S^{d},{ }^{*}, \tau\right)$ is a symmetric involutive semigroup, and the corresponding positive domain $P$ in $S^{d}$ is

$$
P=\left\{\left(s_{2}^{*} s_{1}, s_{1}^{*} s_{2}\right):\left(s_{1}, s_{2}\right) \in S^{d}\right\} .
$$

The semigroup $S^{d}$ acts on $S$ from the right by $s .\left(s_{1}, s_{2}\right):=s_{2}^{*} s s_{1}$. Let $\eta: S \rightarrow$ $S^{d}, s \mapsto\left(s, s^{*}\right)$. Then

$$
\eta\left(s .\left(s_{1}, s_{2}\right)\right)=\left(s_{2}^{*} s s_{1}, s_{1}^{*} s^{*} s_{2}\right)=\left(s_{2}^{*}, s_{1}^{*}\right) \eta(s)\left(s_{1}, s_{2}\right)=\tau\left(s_{1}, s_{2}\right)^{*} \eta(s)\left(s_{1}, s_{2}\right)
$$

and $P=\eta(S S)$, where $S S:=\left\{s_{1} s_{2}: s_{1}, s_{2} \in S\right\}$. Hence we can identify the subsemigroup $S S$ with $P$ as a right $S^{d}$-space. Under this identification the canonical map $q: S^{d} \rightarrow S$ is given by

$$
q\left(s_{1}, s_{2}\right)=s_{2}^{*} s_{1} .
$$

In the special case where $G=S$ is a group and $g^{*}=g^{-1}$, we obtain the realization of $G$ as a symmetric space of the group $G \times G$.

(b) A variation of (a) can be carried out in even greater generality. Let $S$ be any semigroup. Then the semigroup $S^{c}:=S \times S^{o p}$, where $S^{o p}$ is the set $S$ with the multiplication $s_{1} \cdot s_{2}=s_{2} s_{1}$, is an involutive semigroup with respect to $\left(s_{1}, s_{2}\right)^{*}:=\left(s_{2}, s_{1}\right)$, because

$$
\left(\left(s_{1}, s_{2}\right) \cdot\left(t_{1}, t_{2}\right)\right)^{*}=\left(s_{1} t_{1}, t_{2} s_{2}\right)^{*}=\left(t_{2} s_{2}, s_{1} t_{1}\right)=\left(t_{1}, t_{2}\right)^{*}\left(s_{1}, s_{2}\right)^{*} .
$$

Now $\left(S^{c},{ }^{*}, \mathrm{id}_{S^{c}}\right)$ is a symmetric involutive semigroup and $S^{c}$ acts on $S$ from the right by $s .\left(s_{1}, s_{2}\right):=s_{2} s s_{1}$. Let $\eta: S \rightarrow S^{c}, s \mapsto(s, s)$ denote the diagonal map. Then

$$
\eta\left(s .\left(s_{1}, s_{2}\right)\right)=\left(s_{2} s s_{1}, s_{2} s s_{1}\right)=\left(s_{2}, s_{1}\right) \eta(s)\left(s_{1}, s_{2}\right)=\left(s_{1}, s_{2}\right)^{*} \eta(s)\left(s_{1}, s_{2}\right) .
$$


As in (a), we can identify the subsemigroup $S S \subseteq S$ with the positive domain associated to $\left(S^{c},{ }^{*}, \operatorname{id}_{S^{c}}\right)$.

\section{Positive Definite Spherical FunCtions on positive domains}

We recall the basic concepts concerning positive definite kernels and functions. For a detailed discussion we refer to [Ne99, Ch. I-IV] (cf. also [Ar50]). Let $X$ be a set. A function $K: X \times X \rightarrow \mathbb{C}$ is called a positive definite kernel if for each finite subset $\left\{x_{1}, \ldots, x_{n}\right\} \subseteq P$ the matrix $\left(K\left(x_{i}, x_{j}\right)\right)_{i, j=1, \ldots, n}$ is positive semidefinite. This condition is equivalent to the following one (cf. [Ne99, Thm. I.1.4]). There exists a Hilbert space $\mathcal{H} \subseteq \mathbb{C}^{X}$ with continuous point evaluations represented by the functions $K_{x}: y \mapsto K(y, x)$, i.e., $f(x)=\left\langle f, K_{x}\right\rangle$ for all $f \in \mathcal{H}$ and $x \in X$. Then $K$ is called the reproducing kernel of $\mathcal{H}$, and since $\mathcal{H}$ is, as a subspace of $\mathbb{C}^{X}$, uniquely determined by $K$, we put $\mathcal{H}_{K}:=\mathcal{H}$. The dense subspace of $\mathcal{H}_{K}$ spanned by the elements $K_{x}, x \in X$, is denoted $\mathcal{H}_{K}^{0}$. We write $\mathcal{P}\left(X^{2}\right)$ for the space of positive definite kernels on $X$. For $K_{1}, K_{2} \in \mathbb{C}^{X \times X}$ we write $K_{1}<<K_{2}$ if and only if $K_{2}-K_{1} \in \mathcal{P}\left(X^{2}\right)$. This defines a partial order on $\mathbb{C}^{X \times X}$, since a kernel which is at the same time positive and negative definite vanishes. We say that $K_{1}$ dominates $K_{2}$, and write $K_{2} \prec K_{1}$, if there exists $\lambda>0$ with $\lambda K_{1}-K_{2} \in \mathcal{P}\left(X^{2}\right)$, i.e., $K_{2}<<\lambda K_{1}$.

If $S$ is an involutive semigroup, then a function $\varphi$ on $S$ is said to be positive definite if the kernel given by $K_{\varphi}(s, t):=\varphi\left(s t^{*}\right)$ is a positive definite kernel.

If we have a right action $X \times S \rightarrow X$ of an involutive semigroup $S$ on the set $X$, then a function $K: X \times X \rightarrow \mathbb{C}$ is called invariant if

$$
K(x . s, y)=K\left(x, y . s^{*}\right)
$$

for $x, y \in X$ and $s \in S$. Here the terminology is inspired by the group case, where it means that $K(x . g, y . g)=K(x, y)$ for $g \in G$.

Let $\mathcal{H}^{0}$ be a pre-Hilbert space and $\mathcal{H}$ its Hilbert space completion. We write $B_{0}\left(\mathcal{H}^{0}\right)$ for the set of all linear operators $A: \mathcal{H}^{0} \rightarrow \mathcal{H}^{0}$ for which there exists an operator $A^{\sharp}: \mathcal{H}^{0} \rightarrow \mathcal{H}^{0}$ with

$$
\langle A . v, w\rangle=\left\langle v, A^{\sharp} . w\right\rangle
$$

for all $v, w \in \mathcal{H}^{0}$. Note that such an operator $A^{\sharp}$ is uniquely determined by this property. We recall from [Ne99, Lemma II.3.2] that $B_{0}\left(\mathcal{H}^{0}\right)$ is an involutive semigroup with respect to the involution $A \mapsto A^{\sharp}$ and composition of operators.

If $(S, *)$ is an involutive semigroup, then a morphism of involutive semigroups $\pi: S \rightarrow B_{0}\left(\mathcal{H}^{0}\right)$ is called an hermitian representation of $S$ on the pre-Hilbert space $\mathcal{H}^{0}$. A representation is an hermitian representation $(\pi, \mathcal{H})$, where $\mathcal{H}$ is a Hilbert space. If, in addition, $S$ carries a topology, then a representation is said to be continuous if all the functions $S \rightarrow \mathbb{C}, s \mapsto\langle\pi(s) . v, v\rangle, v \in \mathcal{H}$, are continuous.

In this section let $\left(S,{ }^{*}\right)$ always denote an involutive semigroup. Let $X$ be a right $S$-space. According to [Ne99, Prop. II.4.3], a positive definite kernel $K$ on $X$ is invariant if and only if the action of $S$ on $\mathbb{C}^{X}$ given by $(s . f)(x):=f(x . s)$ leaves the pre-Hilbert space $\mathcal{H}_{K}^{0}$ invariant and defines on this space an hermitian representation $\left(\pi_{K}, \mathcal{H}_{K}^{0}\right)$. An invariant positive definite kernel $K$ is said to be exponentially bounded if all the operators $\pi_{K}(s), s \in S$, on $\mathcal{H}_{K}^{0}$ are bounded. We write $\mathcal{P}\left(X^{2}\right)_{\text {inv }}$, resp. $\mathcal{P}\left(X^{2}\right)_{\text {exp }}$, for the cone of invariant resp. exponentially bounded invariant positive definite kernels on $X$. If a kernel $K$ comes from a positive definite function $\varphi$ via $K(s, t)=\varphi\left(s t^{*}\right)$, then we denote the reproducing kernel space $\mathcal{H}_{K}$ 
by $\mathcal{H}_{\varphi}$ and the corresponding representation of $S$ by $\pi_{\varphi}$. An exponentially bounded positive definite function $\varphi$ on $S$ is called spherical if the representation of $S$ on $\mathcal{H}_{\varphi}$ is irreducible.

Lemma 2.1. For $\mathcal{P}\left(X^{2}\right)_{\text {exp }}$ the representation $\pi_{K}$ associated to an exponentially bounded invariant kernel is irreducible if and only if $\mathbb{R}^{+} K$ is an extremal ray in the convex cone $\mathcal{P}\left(X^{2}\right)_{\text {exp }}$.

Proof. [Ne99, Cor. II.4.23].

Definition 2.2. Let $\left(S,{ }^{*}, \tau\right)$ be a symmetric involutive semigroup and $P$ the associated positive domain. A kernel $K \in \mathcal{P}\left(P^{2}\right)_{\text {exp }}$ is called pure if the corresponding representation of $S$ on $\mathcal{H}_{K} \subseteq \mathbb{C}^{P}$ is irreducible. We write $\mathcal{P}\left(P^{2}\right)_{\text {pure }}$ for the cone of pure kernels on $P$.

Let $q: S \rightarrow P, s \mapsto \tau(s)^{*} s$ denote the canonical map. A function $\varphi: P \rightarrow \mathbb{C}$ is called a positive definite function on $P$ if $\varphi\left(q\left(s t^{*}\right)\right)=\overline{\varphi\left(q\left(t s^{*}\right)\right)}$ for all $s, t \in S$ and

$$
K(q(s), q(t)):=\varphi\left(q\left(s t^{*}\right)\right)
$$

defines a positive definite kernel on $P$. If, in addition, (2.1) defines a pure kernel, then $\varphi$ is called the associated positive definite spherical function on $P$. Note here that the right hand side can be written as $\varphi\left(q(s) \cdot t^{*}\right)=\overline{\varphi\left(q(t) \cdot s^{*}\right)}$, hence depends only on the image of $s$, resp. $t$, under $q$, so that (2.1) really defines a function on $P \times P$.

It is clear that if $K$ is a positive definite kernel on $P$ with $(2.1)$, then $\varphi\left(q(s) \cdot t^{*}\right)=$ $\overline{\varphi\left(q(t) \cdot s^{*}\right)}$ holds automatically.

One reason for considering kernels instead of functions in the semigroup setting is that in general the positive domain $P \subseteq S$ has nothing comparable to a base point if $S$ does not have a unit element. This means that we cannot write $P$ as an $S$-orbit of a point $x_{0} \in P$. Nevertheless, in all cases of interest, it turns out that invariant kernels are defined by functions as in Definition 2.2.

Proposition 2.3. Let $\left(S,{ }^{*}, \tau\right)$ be a symmetric involutive semigroup and $P$ the associated positive domain.

(i) Let $K_{1}$ and $K_{2}$ be two $S$-invariant positive definite exponentially bounded kernels on $P$ which correspond to irreducible representations. If $\mathcal{H}_{K_{1}} \cap \mathcal{H}_{K_{2}} \neq$ $\{0\}$, then $\mathcal{H}_{K_{1}}=\mathcal{H}_{K_{2}}$.

(ii) An irreducible representation of $S$ on a reproducing kernel space $\mathcal{H}$ on $P$ corresponds to at least two non-proportional reproducing kernels if and only if the representation on $\mathcal{H} \oplus \mathcal{H}$ can be realized in a reproducing kernel space on $P$.

Proof. (i) First [Ne99, Rem. I.1.7(a)] shows that $\mathcal{H}_{K_{1}+K_{2}}=\mathcal{H}_{K_{1}}+\mathcal{H}_{K_{2}}$. Let

$$
\mathcal{K}:=\left\{(f,-f) \in \mathcal{H}_{K_{1}} \oplus \mathcal{H}_{K_{2}}: f \in \mathcal{H}_{K_{1}} \cap \mathcal{H}_{K_{2}}\right\}
$$

be the kernel of the addition map $\mathcal{H}_{K_{1}} \oplus \mathcal{H}_{K_{2}} \rightarrow \mathcal{H}_{K_{1}+K_{2}}$. Then

$$
\mathcal{H}_{K_{1}} \oplus \mathcal{H}_{K_{2}} \cong \mathcal{K} \oplus \mathcal{H}_{K_{1}+K_{2}}
$$

and since the representations on the subspaces $\mathcal{H}_{K_{i}}$ are irreducible, it follows that the representations on $\mathcal{K}$ and $\mathcal{H}_{K_{1}+K_{2}}$ are irreducible. We conclude in particular that $K_{1}+K_{2}$ is an extremal ray in the cone of exponentially bounded positive definite $S_{C}$-invariant kernels on $P_{C}$ ([Ne99, Cor. II.4.23]). But $K_{1}+K_{2}$ dominates 
$K_{1}$ and $K_{2}$, so we find that $K_{1}, K_{2} \in \mathbb{R}^{+}\left(K_{1}+K_{2}\right)$. This means in particular that $K_{1}$ and $K_{2}$ are multiples of each other.

(ii) The "only if" part is an immediate consequence of (i). For the converse we note that [Ne99, Prop. I.1.8] and its proof show that the kernel $K$ with $\mathcal{H} \oplus \mathcal{H}=\mathcal{H}_{K}$ can be written in the form $K=K_{1}+K_{2}$ with $K_{1}, K_{2}$ both $S_{C}$-invariant kernels on $P$ which satisfy $\mathcal{H}_{K_{i}} \cong \mathcal{H}$. Since $\mathcal{H} \oplus\{0\} \neq\{0\} \oplus \mathcal{H}$, the two kernels cannot be proportional.

Remark 2.4. In the situation of Example 1.2 let $D \cong \pi_{1}(H) \subseteq \widetilde{H}$ denote the kernel of the covering map $\widetilde{H} \rightarrow H$. Then $D$ is central in $\widetilde{H}$, and the action of $\widetilde{H}$ on $\mathfrak{q}$ factors over $H$. Hence $D$ acts trivially on the cone $C$, and therefore $D$ is a central subgroup of $\widetilde{\Gamma}(C)$. We conclude for $s \in \widetilde{\Gamma}(C)$ and $d \in D$ that

$$
q(s) . d=d^{*} q(s) d=d^{*} s^{*} s d=d^{-1} s^{*} s d=s^{*} s d^{-1} d=s^{*} s=q(s) .
$$

Thus $D$ acts trivially on $P_{C}$, so that it also acts trivially on the Hilbert spaces $\mathcal{H}_{K}$ for any positive definite invariant kernel $K$ on $P_{C}$.

The next two examples expand on Example 1.4 and will show up again in the study of holomorphic spherical functions in Section 6 .

Example 2.5. (a) Let $\left(S^{d},{ }^{*}, \tau\right)$ be as in Example 1.4(a) and assume that $S=S S$ (this is the case, for instance, if $S$ has an identity element). Let $\varphi$ be a positive definite function on $S$. Then the kernel $K(s, t):=\varphi\left(s t^{*}\right)$ is positive definite and right-invariant. It is invariant with respect to the action of $S^{d}=S \times S$ if and only if

$$
\varphi\left(s_{2}^{*} x s_{1} y^{*}\right)=K\left(x \cdot\left(s_{1}, s_{2}\right), y\right)=K\left(x, y \cdot\left(s_{1}^{*}, s_{2}^{*}\right)\right)=K\left(x, s_{2} y s_{1}^{*}\right)=\varphi\left(x s_{1} y^{*} s_{2}^{*}\right) .
$$

Since $S S=S$ implies that $S S S=S$, this is equivalent to $\varphi(s t)=\varphi(t s)$ for all $s, t \in S$, which means that $\varphi$ is a central function. Note that the condition $S S=S$ also implies that the kernel associated to $\varphi$ via (2.1) using (1.1) agrees with $K$. Thus $\varphi$ is positive definite as a function on the semigroup $S$ if and only if it is positive definite as a function on the positive domain $S$.

(b) In the situation of Example 1.4(b) with $S=S S$ we set $K(s, t):=\varphi(s t)$ for a given function $\varphi$ on $S$. Then

$$
K\left(s .\left(s_{1}, s_{2}\right), t\right)=\varphi\left(s_{2} s s_{1} t\right) \quad \text { and } \quad K\left(s, t .\left(s_{1}, s_{2}\right)^{*}\right)=\varphi\left(s s_{1} t s_{2}\right),
$$

and again the kernel $K$ is invariant if and only if $\varphi$ is central.

Example 2.6. Let $S$ be an involutive semigroup with $S S=S$, and $\pi: S \rightarrow B_{1}(\mathcal{H})$ a representation of $S$ on the Hilbert space $\mathcal{H}$ by trace class operators (cf. [Ne99, Sect. III.3] for an extensive discussion of this class of representations). Consider the symmetric involutive semigroup $\left(S^{d},{ }^{*}, \tau\right)$ associated to $\left(S,{ }^{*}\right)$ as in Example 1.4(a). Then we obtain an involutive representation of $S^{d}$ on the Hilbert space $B_{2}(\mathcal{H})$ of Hilbert-Schmidt operators by

$$
\rho\left(s_{1}, s_{2}\right)(A):=\pi\left(s_{1}\right) A \pi\left(s_{2}^{*}\right)=\pi\left(s_{1}\right) A \pi\left(s_{2}\right)^{*} .
$$

Since $B_{1}(\mathcal{H}) \subseteq B_{2}(\mathcal{H})$, the representation $\pi$ yields a map $\gamma: S \rightarrow B_{2}(\mathcal{H}), s \mapsto \pi(s)^{*}$ satisfying the equivariance condition

$$
\gamma\left(s .\left(s_{1}, s_{2}\right)\right)=\pi\left(s_{2}^{*} s s_{1}\right)^{*}=\pi\left(s_{1}\right)^{*} \gamma(s) \pi\left(s_{2}\right)=\rho\left(s_{1}, s_{2}\right)^{*} \cdot \pi(s) .
$$


Consider the function $\Theta_{\pi}(s):=\operatorname{tr}(\pi(s))$, which we call the character of the representation (cf. [Ne94b]). [Ne99, Prop. III.3.22] and Example 2.6(a) imply that

$$
K(s, t):=\langle\gamma(t), \gamma(s)\rangle=\operatorname{tr}\left(\pi\left(t^{*}\right) \pi(s)\right)=\operatorname{tr}\left(\pi\left(s t^{*}\right)\right)
$$

defines a positive definite $S^{d}$-invariant kernel on $S$. Thus $\Theta_{\pi}$ is a positive definite function on $S$ defining the kernel $K$. We want to see which condition we have to impose on $\pi$ so that $\Theta_{\pi}$ is a spherical function when considered as a function on the positive domain $S$ of $S^{d}$. Note that (cf. (1.1))

$$
\begin{aligned}
\Theta_{\pi}\left(q\left(\left(s_{1}, s_{2}\right)\left(t_{1}, t_{2}\right)^{*}\right)\right) & =\Theta_{\pi}\left(q\left(s_{1} t_{1}^{*}, s_{2} t_{2}^{*}\right)\right)=\Theta_{\pi}\left(t_{2} s_{2}^{*} s_{1} t_{1}^{*}\right) \\
& =\Theta_{\pi}\left(s_{2}^{*} s_{1} t_{1}^{*} t_{2}\right)=K\left(s_{2}^{*} s_{1}, t_{2}^{*} t_{1}\right) \\
& =K\left(q\left(s_{1}, s_{2}\right), q\left(t_{1}, t_{2}\right)\right) .
\end{aligned}
$$

Therefore $\Theta_{\pi}$ is spherical if and only if the representation of $S^{d}$ on the reproducing kernel space $\mathcal{H}_{K}$ is irreducible. This space is $S^{d}$-equivalent to the closed subspace of $B_{2}(\mathcal{H})$ generated by $\pi(S)$ (cf. [Ne99, Prop. III.3.22]).

If the representation $(\pi, \mathcal{H})$ of $S$ on $\mathcal{H}$ is irreducible, then the representation $\left(\pi \otimes \pi^{*}, B_{2}(\mathcal{H})\right)$ of $S^{d}=S \times S$ is also irreducible (cf. [Ne99, Prop. III.3.22(iv)]). Here $\pi^{*}$ denotes the dual representation on the dual space $\mathcal{H}^{*}$ given by $\pi^{*}(s) . \alpha:=$ $\alpha \circ \pi\left(s^{*}\right)$. It follows in particular that the invariant subset $\pi(S)$ is total in $B_{2}(\mathcal{H})$ and that $\Theta_{\pi}$ is a positive definite spherical function on $S$.

Since $\pi(S)$ consists of compact operators which are of trace class, the representation $\pi$ is a discrete sum of irreducible representations $\pi=\sum_{j} n_{j} \pi_{j}$ with finite multiplicities (cf. [Ne99, Thm. III.3.19]). Then $\Theta_{\pi}=\sum_{j} n_{j} \Theta_{\pi_{j}}$. We claim that $\Theta_{\pi}$ is spherical if and only if $\pi=n_{1} \pi_{1}$ holds for an irreducible representation. To see this, we have to show that a non-trivial sum of multiples of irreducible characters is no longer extremal in the cone of exponentially bounded central positive definite functions on $S$ (cf. Lemma 2.1). This follows from the fact that characters of mutually inequivalent representations are linearly independent ([Ne99, Prop. III.3.24]).

\section{The LÜSCHER-MACK CORRESPONDENCE}

We take up the situation of Example 1.2 and write $\mathfrak{g}^{c}:=\mathfrak{h}+i \mathfrak{q} \subseteq \mathfrak{g}_{\mathbb{C}}$ for the dual symmetric Lie algebra. The main assertion of the following theorem is the result that any bounded representation of the involutive semigroup $\widetilde{\Gamma}\left(C^{0}\right)$ on a Hilbert space $\mathcal{H}$ defines a uniquely determined unitary representation of the simply connected group $G^{c}$ with Lie algebra $\mathfrak{g}^{c}$. This is a remarkable fact, because it is not always true that a representation of a Lie algebra $\mathfrak{g}^{c}$ by essentially skew-adjoint operators on a dense subspace of a Hilbert space integrates to a representation of the corresponding simply connected group (cf. [Wa72, p.296]). The following theorem is a substantially improved version of Theorem 9.39 in [HiNe93] (cf. also [LM75]) which no longer needs the assumption that the representation under consideration is a representation by contractions and that it extends to a continuous representation of the closed cone.

Theorem 3.1 (Lüscher and Mack). Let $\rho: \widetilde{\Gamma}\left(C^{0}\right) \rightarrow B(\mathcal{H})$ be a non-degenerate continuous representation of the involutive semigroup $\widetilde{\Gamma}\left(C^{0}\right)$ and $G^{c}$ the simply connected Lie group with $\mathrm{L}\left(G^{c}\right)=\mathfrak{g}^{c}$. Then: 
(a) There exists a continuous unitary representation $\widetilde{\pi}: \widetilde{H} \rightarrow U(\mathcal{H})$ such that $\rho(h s)=\widetilde{\pi}(h) \rho(s)$ for all $s \in \widetilde{\Gamma}\left(C^{0}\right)$.

(b) There exists a continuous unitary representation $\pi: G^{c} \rightarrow U(\mathcal{H})$ such that

$$
d \pi(X)=d \widetilde{\pi}(X) \text { for all } X \in \mathfrak{h}
$$

and

$$
d \pi(i X)=i d \rho(X) \quad \text { for } \text { all } X \in C^{0},
$$

where for $Y \in C^{0}$ we write $d \rho(Y)$ for the self-adjoint generator of the semigroup $t \mapsto \rho(\operatorname{Exp} t Y)$, which is bounded from above.

Proof. (a) [Ne99, Cor. II.4.15] yields a unitary representation $\widetilde{\pi}: \widetilde{H} \rightarrow U(\mathcal{H})$ such that $\rho(h s)=\widetilde{\pi}(h) \rho(s)$ for all $s \in \widetilde{\Gamma}\left(C^{0}\right)$. Since $\rho$ is non-degenerate, the set $\rho\left(\widetilde{\Gamma}\left(C^{0}\right)\right) . \mathcal{H}$, which consists of continuous vectors for $\widetilde{H}$, is total in $\mathcal{H}$. Hence the representation $\widetilde{\pi}$ of $\widetilde{H}$ is continuous.

(b) The Equianalyticity Lemma ([HiNe93, Lemma 9.16], cf. also [Nel70, p.591]) asserts the existence of a dense subspace $\mathcal{H}_{1}$ of $\widetilde{\pi}$-analytic vectors in $\mathcal{H}$, and of a 0-neighborhood $U \subseteq \mathfrak{h}$ such that the power series $e^{d \widetilde{\pi}(X)} \cdot v:=\sum_{m=0}^{\infty} \frac{1}{m !} d \widetilde{\pi}(X)^{m} \cdot v$ converges for every $v \in \mathcal{H}_{1}$ and $X \in U_{\mathbb{C}}:=U+i U \subseteq \mathfrak{h}_{\mathbb{C}}$. Moreover, we have

$$
\widetilde{\pi}(\operatorname{Exp} X) \cdot v=\sum_{m=0}^{\infty} \frac{1}{m !} d \widetilde{\pi}(X)^{m} \cdot v
$$

for all $X \in U, v \in \mathcal{H}_{1}$.

Let $X \in C$. Then $\gamma_{X}:[0, \infty[\rightarrow B(\mathcal{H}), t \mapsto \rho(\operatorname{Exp}(t X))$ is a strongly continuous one-parameter semigroup of self-adjoint operators. Hence [Ne95a, Thm. III.1] and [Ne99, Prop. VI.3.2] yield the existence of a strongly continuous extension

$$
\gamma_{X}: \mathbb{C}_{+}:=\{z \in \mathbb{C}: \operatorname{Re} z \geq 0\} \rightarrow B(\mathcal{H})
$$

which is holomorphic on the open right half plane int $\mathbb{C}_{+}$.

With this information at hand we can now use the arguments given in steps 3 through 5 in the proof of [HiNe93, Thm. 9.39] to complete the proof of Theorem 3.1 .

In the following we say that the representations $\rho$ of $\widetilde{\Gamma}\left(C^{0}\right)$ and $\pi$ of $G^{c}$ are related if $\pi$ is obtained from $\rho$ by the Lüscher-Mack Theorem.

We want to characterize the representations of $G^{c}$ obtained from non-degenerate representations of $\widetilde{\Gamma}\left(C^{0}\right)$.

Example 3.2. Let $G$ be a simple simply connected semisimple Lie group and $\theta$ the Cartan involution on $G$. Then we have $\mathfrak{g}=\mathfrak{k}+\mathfrak{p}$ with $\mathfrak{h}=\mathfrak{k}$ and $\mathfrak{p}=\mathfrak{q}$. The cone $C=\mathfrak{p}$ is regular and $\widetilde{\Gamma}\left(C^{0}\right)=G=K \exp \left(C^{0}\right)$, where $K=\exp \mathfrak{k}$. A representation $\rho: G \rightarrow B(\mathcal{H})$ is a weakly continuous map such that the operators in $\rho(K)$ are unitary and the operators in $\rho(\exp \mathfrak{p})$ are selfadjoint.

Here $G^{c}$ is the simply connected compact group with Lie algebra $\mathfrak{g}^{c}=\mathfrak{k}+i \mathfrak{p}$, and the Lüscher-Mack Theorem provides a unitary representation $\pi: G^{c} \rightarrow U(\mathcal{H})$ which is compatible with $\rho$. This has the interesting consequence that, even on infinite dimensional Hilbert spaces, every such representation $\rho$ contains the kernel of the natural map $K \rightarrow G^{c}$ which coincides with the kernel of the natural map $\eta: G \rightarrow G_{\mathbb{C}}$. Hence $\rho$ factors to a representation of the group $\eta(G) \cong G / \eta(G)$ and, 
since $G^{c}$ is compact, the Peter-Weyl Theorem implies that it is a direct Hilbert space sum of irreducible finite dimensional representations.

It follows in particular that all the operators $d \rho(X), X \in \mathfrak{p}$, are bounded; hence, in view of the simplicity of $G$, and the observation that the set of all $X \in \mathfrak{g}^{c}$ for which $i d \pi(X)$ is bounded is an ideal, all operators $i d \pi(X), X \in \mathfrak{g}^{c}$, are bounded. Thus the representation of $G^{c}$ even extends to a holomorphic representation $G_{\mathbb{C}} \rightarrow B(\mathcal{H})$ of the complexified group $G_{\mathbb{C}}$.

Lemma 3.3. If $\rho$ and $\pi$ are related, then the commutants $\rho\left(\widetilde{\Gamma}\left(C^{0}\right)\right)^{\prime}$ and $\pi\left(G^{c}\right)^{\prime}$ in $B(\mathcal{H})$ agree. It follows in particular that a closed subspace of $\mathcal{H}$ is invariant under $\widetilde{\Gamma}\left(C^{0}\right)$ if and only if it is invariant under $G^{c}$, and that $\rho$ is irreducible if and only if $\pi$ is irreducible.

Proof. Since $G^{c}$ is generated by $\exp \mathfrak{h}$ and $\exp \left(i C^{0}\right)$, it suffices to show that an operator commutes with $\rho\left(\operatorname{Exp} C^{0}\right)$ if and only if it commutes with $\pi\left(\exp i C^{0}\right)$. To achieve this, it suffices to show that for $X \in C^{0}$ an operator commutes with $\rho\left(\exp \mathbb{R}^{+} X\right)$ if and only if it commutes with $\pi(\operatorname{Exp} \mathbb{R} i X)$.

For $v, w \in \mathcal{H}$ and $A \in B(\mathcal{H})$ consider the map

$$
\mathbb{C}_{+} \rightarrow \mathcal{H}, z \mapsto\left\langle A \gamma_{X}(z) . v-\gamma_{X}(z) A . v, w\right\rangle,
$$

which is continuous and on int $\mathbb{C}_{+}$even holomorphic (cf. (3.1)). Hence it vanishes on $\mathbb{R}^{+}$if and only if it vanishes on the boundary $i \mathbb{R}$ ([HiNe93, Lemma 9.17]). Since $v$ and $w$ are arbitrary, this proves that $A$ commutes with $\gamma_{X}(i \mathbb{R})$ if and only if it commutes with $\gamma_{X}\left(\mathbb{R}^{+}\right)$. But Theorem 3.1 implies $\pi(\exp i t X)=\gamma_{X}(i t)$, and hence $\rho(\operatorname{Exp} t X)=\gamma_{X}(t)$ proves the claim.

For any Lie algebra $\mathfrak{g}$ we denote by $\operatorname{comp}(\mathfrak{g})$ the set of compact elements in $\mathfrak{g}$, i.e., those elements $X$ for which the closure of the group $e^{\mathbb{R} a d X} \subseteq \operatorname{Aut}(\mathfrak{g})$ is compact. A subalgebra consisting of compact elements is called compactly embedded. Let $\mathfrak{t} \subseteq \mathfrak{g}$ be a compactly embedded Cartan subalgebra. The existence of a compactly embedded Cartan subalgebra is equivalent to the existence of interior points of $\operatorname{comp}(\mathfrak{g})([$ HHL89, A.2.27]). Moreover, an element $X \in \mathfrak{t}$ is contained in int $\operatorname{comp}(\mathfrak{g})$ if and only if $\mathfrak{g}_{X}:=\operatorname{ker} a d X \subseteq \mathfrak{k}$ ([HHL89, A.2.25]). It follows in particular that all regular elements in $\mathfrak{t}$ are contained in $\operatorname{int}(\operatorname{comp}(\mathfrak{g}))$. Note further that there is a uniquely determined maximal compactly embedded subalgebra $\mathfrak{k}$ containing $\mathfrak{t}$ ([HHL89, A.2.40]).

In the following we will use the root space decomposition

$$
\mathfrak{g}_{\mathbb{C}}=\mathfrak{t}_{\mathbb{C}} \oplus \bigoplus_{\alpha \in \Delta} \mathfrak{g}_{\mathbb{C}}^{\alpha}
$$

associated to the Cartan subalgebra $\mathfrak{t}_{\mathbb{C}}$ of $\mathfrak{g}_{\mathbb{C}}$. A root $\alpha$ is called non-compact if $\mathfrak{g}_{\mathbb{C}}^{\alpha} \nsubseteq \mathfrak{k}_{\mathbb{C}}$. The set of all non-compact roots is denoted by $\Delta_{p}$ and we write $\Delta_{k}$ for the set of compact roots. Let $\Delta^{+} \subseteq \Delta$ be a positive system. It is called $\mathfrak{k}$-adapted if $\Delta_{p}^{+}=\Delta^{+} \cap \Delta_{p}$ is invariant under the action of the Weyl group for the pair $(\mathfrak{k}, \mathfrak{t})$.

For a complex $\mathfrak{g}_{\mathbb{C}}$-module $V$ and $\lambda \in \mathfrak{t}_{\mathbb{C}}^{*}$ we write

$$
V^{\lambda}:=\left\{v \in V:\left(\forall X \in \mathfrak{t}_{\mathbb{C}}\right) X . v=\lambda(X) v\right\}
$$

for the weight space of weight $\lambda$, and $\lambda$ is said to be a weight of $V$ is $V^{\lambda} \neq\{0\}$. We write $\mathcal{P}_{V}$ for the set of weights of $V$. An element $0 \neq v \in V^{\lambda}$ is called a primitive element of $V$ of weight $\lambda$ (with respect to a positive system $\Delta^{+}$) if $\mathfrak{g}_{\mathbb{C}}^{\alpha} \cdot v=\{0\}$

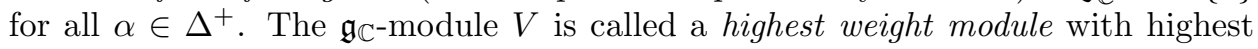


weight $\lambda$ (with respect to $\Delta^{+}$) if it is generated by a primitive element of weight $\lambda$. Finally, the $\mathfrak{g}_{\mathbb{C}}$ module $V$ is called $\mathfrak{k}$-finite if it consists of $\mathfrak{k}$-finite vectors, i.e., if $\operatorname{dim} \mathcal{U}\left(\mathfrak{k}_{\mathbb{C}}\right) \cdot v<\infty$ for all $v \in V$.

In the following we will write $K=\exp \mathfrak{k}$ for the analytic subgroup $K$ of $G$ with Lie algebra $\mathfrak{k}$. For a unitary representation of $G$ on the Hilbert space $\mathcal{H}$ and we denote the space of $K$-finite vectors in $\mathcal{H}$ by $\mathcal{H}^{K}$. Similarly, $\mathcal{H}^{K, \infty}$ and $\mathcal{H}^{K, \omega}$ denote the smooth and analytic $K$-finite vectors in $\mathcal{H}$, respectively.

A unitary representation $(\pi, \mathcal{H})$ of $G$ is called a unitary highest weight representation if the $\mathfrak{g}_{\mathbb{C}}$-module $\mathcal{H}^{K, \infty}$ of $K$-finite smooth vectors in $\mathcal{H}$ is a highest weight module.

The following theorem is a generalization of Theorem III.6 in [Ne94b].

Theorem 3.4. Let $(\pi, \mathcal{H})$ be a continuous unitary representation of the connected Lie group $G, \mathfrak{t} \subseteq \mathfrak{g}$ a compactly embedded Cartan subalgebra, $\mathfrak{k} \supseteq \mathfrak{t}$ a maximal compactly embedded subalgebra, $K=\exp \mathfrak{k}, \Delta^{+}$a $\mathfrak{k}$-adapted positive system, and $X \in \operatorname{int}\left(i \Delta_{p}^{+}\right)^{\star} \subseteq i$ t. Then $(1) \Rightarrow(2)$ holds for the following assertions:

(1) The operator $i d \pi(X)$ is bounded from above and $\mathcal{H}^{K} \neq\{0\}$.

(2) $\mathcal{H}^{K, \omega}$ contains a primitive element with respect to $\Delta^{+}$.

If (2) is satisfied, then the following are equivalent:

(3) $\pi$ is an irreducible representation.

(4) $\mathcal{H}^{K, \infty}$ is a highest weight module with respect to $\Delta^{+}$, and $\mathcal{H}^{K}$ is dense.

(5) $\mathcal{H}^{K, \infty}$ is an irreducible highest weight module with respect to $\Delta^{+}$, and $\mathcal{H}^{K}$ is dense.

If (5) is satisfied, then $\mathcal{H}^{K}=\mathcal{H}^{K, \infty}$ consists of analytic vectors and (1) is satisfied.

Proof. $(1) \Rightarrow(2)$ : We assume that the subspace $\mathcal{H}^{K}$ of $K$-finite vectors in non-zero. In view of [Ne94b, Prop. III.4], the space $V:=\mathcal{H}^{K, \omega}$ of analytic $K$-finite vectors in dense in $\mathcal{H}^{K}$, so that in particular $V \neq\{0\}$. Since $V$ is the intersection of two

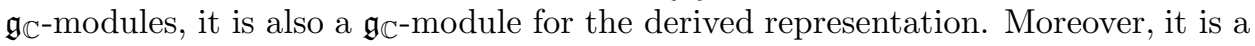
semisimple $\mathfrak{t}_{\mathbb{C}}$-module. Hence it has a weight decomposition

$$
V=\bigoplus_{\mu \in \mathcal{P}_{V}} V^{\mu}
$$

where $\mathcal{P}_{V} \subseteq i \mathfrak{t}^{*} \subseteq \mathfrak{t}_{\mathbb{C}}^{*}$ is a non-empty set.

Next we use the assumption that $i d \pi(X)$ is bounded from above to see that

$$
m:=\sup \left\langle i \mathcal{P}_{V}, X\right\rangle<\infty .
$$

Pick $\mu \in \mathcal{P}_{V}$ such that

$$
i \mu(X)>m-\min \left\{i \alpha(X): \alpha \in \Delta_{p}^{+}\right\} .
$$

Then $i(\mu+\alpha)(X)>m$ holds for all $\alpha \in \Delta_{p}^{+}$. Let $\mathfrak{p}^{+}:=\sum_{\alpha \in \Delta_{p}^{+}} \mathfrak{g}_{\mathbb{C}}^{\alpha}$. Then

$$
\{0\} \neq V^{\mu} \subseteq V_{+}:=\left\{w \in V: \mathfrak{p}^{+} . w=\{0\}\right\} .
$$

Since $\mathfrak{p}^{+}$is an ideal in the subalgebra $\mathfrak{p}^{+}+\mathfrak{k}_{\mathbb{C}}$ of $\mathfrak{g}_{\mathbb{C}}$ (here we need that $\Delta^{+}$is $\mathfrak{k}$-adapted), the space $V_{+}$is a $\mathfrak{k}_{\mathbb{C}}$-submodule of $V$.

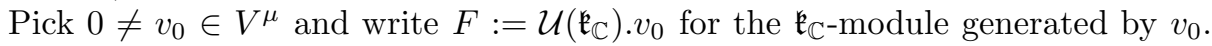
Since this module is finite dimensional, it contains a primitive element $v$ for $\mathfrak{k}_{\mathbb{C}}$ with respect to $\Delta_{k}^{+}$. Then $v \in V_{+}$implies that $v$ is also a primitive element for $\mathfrak{g}_{\mathbb{C}}$ with respect to $\Delta^{+}$, and this proves (2). 
The proof for the equivalence between (3), (4), (5) and the conclusion from (5) require no modification of the proof given in [Ne94b, Thm. III.6].

Definition 3.5. Let $\pi$ be a unitary represention of $G^{c}$ in the Hilbert space $\mathcal{H}$. Then $\Psi: \mathbb{P}\left(\mathcal{H}^{\infty}\right) \rightarrow\left(\mathfrak{g}^{c}\right)^{*}$ defined by

$$
\Psi([v])(X)=\frac{\langle i d \pi(X) \cdot v, v\rangle}{\langle v, v\rangle},
$$

where $\mathbb{P}\left(\mathcal{H}^{\infty}\right)$ is the projective space associated to $\mathcal{H}^{\infty}$, is called the moment map of $\pi$ and

$$
I_{\pi}:=\overline{\operatorname{conv} \Psi\left(\mathbb{P}\left(\mathcal{H}^{\infty}\right)\right)} \subseteq\left(\mathfrak{g}^{c}\right)^{*},
$$

is called the moment set. The not necessarily closed invariant cone

$$
W_{\pi}:=\left\{X \in \mathfrak{g}^{c}: \sup \left\langle X, I_{\pi}\right\rangle<\infty\right\}
$$

will be referred to as the associated cone for $\pi$.

Now suppose that $\rho$ is a continuous representation of $S_{C}$ and $\pi$ is related to $\rho$. Then [Ne99, Prop. X.1.6] says that for $X \in \mathfrak{g}^{c}$ we have

$$
\sup \operatorname{Spec}(i d \pi(X))=\sup \left\langle X, I_{\pi}\right\rangle \text {. }
$$

Then the fact that the operators $d \rho(X)=i d \pi(-i X)$ for $X \in C^{0}$ are bounded from above shows that $-i C^{0} \subseteq W_{\pi}$, i.e., $C^{0} \subseteq i W_{\pi}$.

For the proof of the following theorem we will need Lemma A.1 from Appendix A.

Theorem 3.6. Let $\mathfrak{g}$ be a Lie algebra, $(\pi, \mathcal{H})$ an irreducible continuous unitary representation of a connected Lie group $G$ with $\mathrm{L}(G)=\mathfrak{g}$, and suppose that there exists $X \in \operatorname{int} \operatorname{comp}(\mathfrak{g})$ such that the operator $i d \pi(X)$ is bounded from above. Then

(1) $\pi$ is a unitary highest weight representation with respect to a compactly embedded Cartan subalgebra and an associated positive system of roots, and

(2) the cone $W_{\pi}$ consists of all those elements $X \in \mathfrak{g}$ for which the operator $i d \pi(X)$ is bounded from above, and has interior points.

Proof. First we note that we may w.l.o.g. assume that the group $G$ is simply connected.

(1) Let $\mathfrak{b}:=\operatorname{ker} d \pi$. Then $\mathfrak{b} \subseteq \mathfrak{g}$ is an ideal. We write $\psi: \mathfrak{g} \rightarrow \mathfrak{g} / \mathfrak{b}$ for the quotient map and $B:=(\operatorname{ker} \pi)_{0}$ for the identity component of the kernel. Then the representation $\pi$ of $G$ factors to a representation $\bar{\pi}$ of $G / B$ on $\mathcal{H}$. Moreover $\psi(\operatorname{comp}(\mathfrak{g})) \subseteq \operatorname{comp}(\mathfrak{g} / \mathfrak{b})$ implies that $\psi(X) \in \psi(\operatorname{int} \operatorname{comp}(\mathfrak{g})) \subseteq \operatorname{int} \operatorname{comp}(\mathfrak{g} / \mathfrak{b})$, and $i d \bar{\pi}(\psi(X))=i d \pi(X)$ is bounded from above. Hence all the assumptions that we have made for $G$ are satisfied for the group $G / B$. Thus we may w.l.o.g. assume that the representation $\pi$ has discrete kernel.

In view of the assumption that $\pi$ has discrete kernel, the moment set $I_{\pi} \subseteq \mathfrak{g}^{*}$ satisfies $I_{\pi}^{\perp}=\operatorname{ker} d \pi=\{0\}$. Moreover (3.2) shows that the associated cone $W_{\pi}$ for $\pi$ consists precisely of all those elements $X \in \mathfrak{g}$ for which $i d \pi(X)$ is bounded from above. Hence $W_{\pi}$ contains the element $X$. So the ideal $\mathfrak{a}:=W_{\pi}-W_{\pi}$ generated by $W_{\pi}$ intersects int $\operatorname{comp}(\mathfrak{g})$.

We write $p_{\mathfrak{a}}: \mathfrak{g}^{*} \rightarrow \mathfrak{a}^{*}$ for the restriction map and put $J_{\pi}:=\overline{p_{\mathfrak{a}}\left(I_{\pi}\right)}$. Then $J_{\pi}$ is a closed convex invariant subset in $\mathfrak{a}^{*}$ and

$$
W_{\pi}=\left\{Y \in \mathfrak{a}: \sup \left\langle Y, J_{\pi}\right\rangle<\infty\right\} .
$$


Since $W_{\pi}$ has interior points in $\mathfrak{a}$, we conclude that the closed convex set $J_{\pi}$ contains no affine subspaces of positive dimension. Moreover $J_{\pi}^{\perp}=I_{\pi}^{\perp} \cap \mathfrak{a}=\{0\}$. Therefore [Ne99, Lemma VII.3.1] shows that $\mathfrak{a}$ is admissible, and then $\mathfrak{g}$ is also admissible by Lemma A.1 (Appendix A).

Let $\mathfrak{g}=\mathfrak{u} \rtimes \mathfrak{l}$ denote the decomposition of $\mathfrak{g}$ where $\mathfrak{u}$ is the nilradical and $\mathfrak{l}$ is a reductive $\mathfrak{t}$-invariant subalgebra. We distinguish two cases.

Case 1: The Lie algebra $\mathfrak{g}$ is reductive, i.e., $\mathfrak{g}=\mathfrak{l}$. Then $G$ is a (CA) group, so that, according to [Ne94b, Prop. III.2], the space $\mathcal{H}^{K}$ of $K$-finite vectors in $\mathcal{H}$ is dense.

Case 2: The Lie algebra $\mathfrak{g}$ is not reductive, i.e., $\mathfrak{u} \neq\{0\}$. We write $G=U \rtimes L$ for the semidirect decomposition of $G$ corresponding to the decomposition, $\mathfrak{g}=\mathfrak{u} \rtimes \mathfrak{l}$, and recall that $\operatorname{dim} \mathfrak{z}(\mathfrak{g}) \geq 1$ ([HiNe93, Thm. 7.15]). On the other hand, $\operatorname{dim} \mathfrak{z}(\mathfrak{g}) \leq 1$ by Schur's Lemma since $\pi$ is irreducible and has discrete kernel. Thus $\operatorname{dim} \mathfrak{z}(\mathfrak{g})=1$.

Let $\Delta^{+} \subseteq \Delta$ be a $\mathfrak{k}$-adapted positive system such that the cone

$$
C_{\text {min }}=\operatorname{cone}\left\{i\left[\overline{X_{\alpha}}, X_{\alpha}\right]: X_{\alpha} \in \mathfrak{g}_{\mathbb{C}}^{\alpha}, \alpha \in \Delta_{p}^{+}\right\} \subseteq \mathfrak{t}
$$

is pointed (cf. [HiNe93, Thm. 7.26] and [Ne94a, Def. II.18]). Then $C_{m i n, z}:=$ $C_{\min } \cap \mathfrak{z}(\mathfrak{g}) \subseteq \mathfrak{z}(\mathfrak{g})$ is a halfline and we may w.l.o.g. assume that the central character $\chi: Z(G) \rightarrow \mathrm{S}^{1}$ defined by $\pi(g)=\chi(g) e$ for all $g \in Z(G)$ satisfies $-i d \chi(e) \in$ $\operatorname{int} C_{\min , \mathfrak{z}}^{\star}$. Thus $\pi$ is a representation of type $\chi$ which is admissible with respect to $\Delta^{+}$in the sense of [Ne96], and Theorem III.2 in [Ne96] shows that the space $\mathcal{H}^{K}$ of $K$-finite vectors in $\mathcal{H}$ is dense.

Now Theorem 3.4 applies in both cases, and shows that $\pi$ is a unitary highest weight representation of $G$ with respect to a positive system $\widetilde{\Delta}^{+}$chosen in such a way that $i \alpha(X)>0$ for all $\alpha \in \widetilde{\Delta}_{p}^{+}$.

(2) Let $W_{\max } \subseteq \mathfrak{g}$ be the uniquely determined invariant closed convex cone with $W_{\text {max }} \cap \mathfrak{t}=\left(i \Delta_{p}^{+}\right)^{\star}$ (cf. [HiNe93, $\left.\S 7.2\right]$ ). Then we use [Ne96, Prop. IV.2] to see that for $Y \in W_{\max }^{0}$, the operator $i d \pi(Y)$ is bounded from above. This means that $W_{\max }^{0} \subseteq W_{\pi}$, and we see in particular that $W_{\pi}$ is generating.

Example 3.7. We consider the special case where $\mathfrak{g}=\mathfrak{h}_{\mathbb{C}}$ is the complexification of the admissible Lie algebra $\mathfrak{h}$ and the involution $\tau$ is complex conjugation (cf. Definition 1.3).

First we exploit the structure of the relevant Lie algebras. We identify $\mathfrak{g}_{\mathbb{C}}=\left(\mathfrak{h}_{\mathbb{C}}\right)_{\mathbb{C}}$ with $\mathbb{C} \otimes_{\mathbb{R}} \mathfrak{h}_{\mathbb{C}}=\mathbb{C} \otimes_{\mathbb{R}} \mathfrak{g}$ and claim that $\mathfrak{g}_{\mathbb{C}} \cong \mathfrak{h}_{\mathbb{C}} \oplus \overline{\mathfrak{h}_{\mathbb{C}}}$. In fact, we define two mappings

$$
\eta_{ \pm}: \mathfrak{h}_{\mathbb{C}} \rightarrow \mathfrak{g}_{\mathbb{C}}, \quad X \mapsto \frac{1}{2}(1 \otimes X \pm i \otimes i X) .
$$

Then [KrNe96, Lemma I.10] shows that

$$
\mathfrak{g}_{\mathbb{C}} \cong \eta_{-}\left(\mathfrak{h}_{\mathbb{C}}\right) \oplus \eta_{+}\left(\mathfrak{h}_{\mathbb{C}}\right) \cong \mathfrak{h}_{\mathbb{C}} \oplus \overline{\mathfrak{h}_{\mathbb{C}}} .
$$

Note that $i(X, Y)=(i X,-i Y)$ holds in $\mathfrak{g}_{\mathbb{C}}$. It is clear that in this representation the complex conjugation $\sigma_{\mathfrak{g}}$ with respect to $\mathfrak{g}$ acts by $\sigma_{\mathfrak{g}}(X, Y)=(Y, X)$, the complex linear extension $\tau$ of the involution $\tau$ on $\mathfrak{h}_{\mathbb{C}}$ by $\tau(X, Y)=(\tau . Y, \tau . X)$, and the complex conjugation $\sigma=\tau \circ \sigma_{\mathfrak{g}}$ with respect to $\mathfrak{g}^{c}$ by $\sigma(X, Y)=(\tau . X, \tau . Y)$. The fixed point set of this involution is

$$
\mathfrak{g}^{c} \cong\{(X, Y): X, Y \in \mathfrak{h}\}=\mathfrak{h} \oplus \mathfrak{h},
$$

whereas $\mathfrak{g} \cong \mathfrak{h}_{\mathbb{C}}$ is embedded as the diagonal

$$
\Delta_{\mathfrak{h}_{\mathbb{C}}}=\left\{(X, X): X \in \mathfrak{h}_{\mathbb{C}}\right\} \subseteq \mathfrak{h}_{\mathbb{C}} \oplus \overline{\mathfrak{h}_{\mathbb{C}}} .
$$


Now let $C \subseteq \mathfrak{q}=i \mathfrak{h}$ be a generating hyperbolic $\widetilde{H}$-invariant cone, i.e., $i C$ is a generating invariant cone in the Lie algebra $\mathfrak{h}$ and for each $X \in C^{0}$ the operator $\operatorname{ad} X$ is diagonalizable over $\mathbb{R}$, i.e., $i C^{0} \subseteq \operatorname{comp}(\mathfrak{g})$. We put $S_{C}:=\widetilde{\Gamma}\left(\mathfrak{h},-i C^{0}\right) \cong$ $\widetilde{H} \operatorname{Exp}\left(C^{0}\right)$, where $\widetilde{H}$ is the associated simply connected group. We consider the invariant cone $W:=(-i C) \oplus i C \subseteq \mathfrak{h} \oplus \mathfrak{h}$. Then $i W=C \oplus C$ implies that $i W \cap \mathfrak{q}=$ $\Delta_{C}=\{(X, X): X \in C\}$, which corresponds to the cone $C$ under the diagonal embedding of $\mathfrak{g}=\mathfrak{h}_{\mathbb{C}}$. Let $G_{\mathbb{C}}$ denote any complex Lie group with Lie algebra $\mathfrak{g}_{\mathbb{C}}$. The semigroup corresponding to $W$ is the universal covering of the subsemigroup of $G_{\mathbb{C}}$ generated by the exponential image of $\mathfrak{g}^{c}+i W=\mathfrak{g}^{c}+(C \oplus C)$. Hence $S_{W} \cong S_{C} \times \overline{S_{C}}$, where $\overline{S_{C}}$ denotes the same semigroup as $S_{C}$ endowed with the opposite complex structure, i.e., the mapping $S_{C} \rightarrow \overline{S_{C}}, s \mapsto s^{*}$ is a holomorphic anti-isomorphism.

Let $G^{c}=\widetilde{H} \times \widetilde{H}$ be the simply connected group associated to $\mathfrak{g}^{c}$. Then each unitary highest weight representation $\pi$ of $G^{c}$ is a tensor product $\pi_{1} \otimes \pi_{2}$ of two unitary highest weight representations $\pi_{1}$ and $\pi_{2}$ of the two $\widetilde{H}$-factors. In fact, the highest weight $\lambda$ of the $G^{c}$-representation is of the form $\lambda=\left(\lambda_{1}, \lambda_{2}\right)$ with two weight functionals $\lambda_{1}, \lambda_{2}$ for $\widetilde{H}$. These belong to irreducible highest weight representations of $\widetilde{H}$ whose tensor product again gives an irreducible unitary representation of $G^{c}$ with highest weight $\lambda$, i.e., agrees with the original representation.

The Holomorphic Extension Theorem from Appendix B implies that a representation is related to a continuous representation of the semigroup $S_{C}$ if and only if the operators $i d \pi(X), X \in-i C$, are bounded from above. To make this condition more explicit, let $X \in C$, and let $(X, X) \in \mathfrak{g}_{\mathbb{C}}$ be the corresponding diagonal element. Then $-i(X, X)=(-i X, i X) \in \mathfrak{g}^{c}$ and

$$
i d \pi(-i(X, X))=d \pi(X, X)=d \pi_{1}(X) \otimes 1+1 \otimes d \pi_{2}(X) .
$$

This operator is bounded from above on $\mathcal{H}_{1} \widehat{\otimes} \mathcal{H}_{2}$ if and only if $i d \pi_{j}(X)$ is bounded from above on $\mathcal{H}_{j}$ for $j=1,2$ ([RS72, p.301]). It follows in particular that this implies that the operators

$$
i d \pi(-i X, i Y)=d \pi(X, Y)=d \pi_{1}(X) \otimes 1+1 \otimes d \pi_{2}(Y)
$$

are bounded from above for $X, Y \in C^{0}$. Hence every irreducible continuous representation of the semigroup $S_{C}$ extends in particular to a holomorphic irreducible representation of the semigroup $S_{W}=S_{C} \times \overline{S_{C}}$. Note that the holomorphic representations of $\overline{S_{C}}$ are just the antiholomorphic representations of $S_{C}$. But if $(\pi, \mathcal{H})$ is a holomorphic representation of the semigroup $S_{C}$, then $\left(\pi^{*}, \mathcal{H}^{*}\right)$ is an antiholomorphic representation of $S_{C}$ given by $\pi^{*}(s) . f:=f \circ \pi\left(s^{*}\right)$. Thus every irreducible continuous representation of $S_{C}$ corresponds to a pair of holomorphic irreducible representations of $S_{C}$ (cf. [Ne94b]).

We denote the equivalence classes of continuous and holomorphic representations of $S_{C}$ by ${\widehat{S_{C}}}^{\text {cont }}$ and ${\widehat{S_{C}}}^{\text {holo }}$, respectively. With this notation we formulate the most important conclusions of Example 3.7 as a proposition:

Proposition 3.8. Let $P_{C}$ be an $O l^{\prime}$ shanskiน domain, $S_{C}$ the corresponding semigroup, and $S_{W}=S_{C} \times \overline{S_{C}}$.

(i) We have the following identifications between equivalence classes of representations:

$$
{\widehat{S_{C}}}^{\text {cont }} \cong{\widehat{S_{W}}}^{\text {holo }} \cong{\widehat{S_{C}}}^{\text {holo }} \times{\widehat{S_{C}}}^{\text {antiholo }}
$$


The first identification is via holomorphic extension, whereas the second says that any $\pi \in{\widehat{S_{W}}}^{\text {holo }}$ is of the form $\pi_{1} \otimes \pi_{2}^{*}$ with $\pi_{1}, \pi_{2} \in{\widehat{S_{C}}}^{\text {holo }}$.

(ii) Any $\pi \in{\widehat{S_{W}}}^{\text {holo }}$ can be realized as the representation on the space

$$
B_{2}\left(\mathcal{H}_{2}, \mathcal{H}_{1}\right) \cong \mathcal{H}_{1} \widehat{\otimes} \mathcal{H}_{2}^{*}
$$

given by

$$
\pi\left(s_{1}, s_{2}\right) \cdot A:=\pi_{1}\left(s_{1}\right) A \pi_{2}\left(s_{2}^{*}\right)
$$

with $\pi_{1}, \pi_{2} \in{\widehat{S_{C}}}^{\text {holo }}$.

We remark here that [Ne96] provides a parametrization of ${\widehat{S_{C}}}^{\text {holo }}$ in terms of highest weight representations of $\widetilde{H}$ (cf. also the Holomorphic Extension Theorem in Appendix B).

Corollary 3.9. Let $G^{c}$ and $S_{C}$ be as in Example 3.7, and let the representations $\pi$ of $G^{c}$ and $\rho$ of $S_{C}$ be related and irreducible. Then $\pi$ is a unitary highest weight representation and $W_{\pi} \subseteq \mathfrak{g}^{c}$ contains interior points.

Proof. According to our assumption that $C$ is hyperbolic, the open cone $i C^{0}$ is contained in the interior of $\operatorname{comp}(\mathfrak{h})$, hence in the interior of $\operatorname{comp}\left(\mathfrak{g}^{c}\right)$ (cf. Example 3.7). Since for each $X \in i C^{0}$ the operator $-i d \pi(X)$ is bounded from above, the assertion follows from Theorem 3.6.

Now we find a complete description of the Lüscher-Mack correspondence, at least for irreducible representations in the case of $\mathrm{Ol}^{\prime}$ shanskil spaces.

Corollary 3.10. Let $G^{c}$ and $S_{C}$ be as in Example 3.7. Then a unitary representation $\pi$ of $G^{c}$ is related to an irreducible continuous representation $\rho$ of $S_{C}$ if and only if it is an irreducible highest weight representation whose associated cone in $\mathfrak{g}^{c}$ contains $C^{0}$.

Proof. The "only if" part follows from Corollary 3.9 and the fact that the $d \rho(X)$ for $X \in C^{0}$ are bounded above for any continuous representation of $S_{C}$. The converse follows from the Holomorphic Extension Theorem in Appendix B.

\section{4. $S_{C}$-SPHERICAL REPRESENTATIONS}

Let $S_{C}:=\widetilde{\Gamma}\left(C^{0}\right) \subseteq \widetilde{\Gamma}(C)=\widetilde{H} \operatorname{Exp}(C)$ be as in Example 1.2. We want to describe the set $\mathcal{P}\left(P_{C}\right)_{\text {pure }}$ of positive definite spherical functions on the corresponding positive domain

$$
P_{C}=\left\{s^{*} s: s \in \widetilde{\Gamma}\left(C^{0}\right)\right\}=\operatorname{Exp}\left(C^{0}\right) .
$$

Definition 4.1. An irreducible unitary representation $(\pi, \mathcal{H})$ of $G^{c}$ is called $S_{C^{-}}$ spherical if there exist a related representation $\rho$ of $S_{C}$ on $\mathcal{H}$ and an $\widetilde{H}$-invariant non-zero linear functional $f$ on the dense subspace $\mathcal{H}^{0}:=\operatorname{span}\left(\rho\left(S_{C}\right) . \mathcal{H}\right)$ of $\mathcal{H}$ such that $f \circ \rho(s): \mathcal{H} \rightarrow \mathbb{C}$ is continuous for all $s \in S_{C}$.

Remark 4.2. Let $H^{c}$ be the analytic subgroup of $G^{c}$ with Lie algebra $\mathfrak{h}$. Then $\mathcal{H}^{0}$ is $\pi\left(H^{c}\right)$-invariant, and a linear functional $f$ on $\mathcal{H}^{0}$ is $\widetilde{H}$-invariant if and only if it is $\pi\left(H^{c}\right)$-invariant. 
Proposition 4.3. An irreducible unitary representation $(\pi, \mathcal{H})$ of $G^{c}$ with the related representation $(\rho, \mathcal{H})$ of $S_{C}$ is spherical if and only if there exists a positive definite spherical function $\varphi$ on $S_{C}$ such that

(a) $(\rho, \mathcal{H}) \cong\left(\pi_{\varphi}, \mathcal{H}_{\varphi}\right)$ as representations of $S_{C}$, and

(b) $\varphi$ factors through $q$ to give a positive definite spherical function $\bar{\varphi}$ on $P_{C}$.

Proof. Let us first assume that $(\pi, \mathcal{H})$ is $S_{C}$-spherical and that $f: \mathcal{H}^{0} \rightarrow \mathbb{C}$ is a nonzero $\widetilde{H}$-invariant linear functional such that $f \circ \rho(s)$ is continuous for all $s \in S_{C}$. We define a map $\gamma: S_{C} \rightarrow \mathcal{H}$ by

$$
(f \circ \rho(s))(v)=\langle v, \gamma(s)\rangle
$$

for all $v \in \mathcal{H}$. Then

$$
\langle v, \gamma(s t)\rangle=(f \circ \rho(s t))(v)=(f \circ \rho(s))(\rho(t) \cdot v)=\langle\rho(t) \cdot v, \gamma(s)\rangle=\left\langle v, \rho\left(t^{*}\right) \cdot \gamma(s)\right\rangle
$$

implies that $\gamma(s t)=\rho(t)^{*} \cdot \gamma(s)$ for all $s, t \in S_{C}$. On the other hand, the $\widetilde{H}$ invariance of $f$ means that $\gamma(h s)=\gamma(s)$ for $s \in S_{C}, h \in \widetilde{H}$. Now $K(s, t):=$ $\langle\gamma(t), \gamma(s)\rangle$ defines a right-invariant positive definite kernel on $S_{C}$, and since $S_{C}$ has an approximate identity, there exists a uniquely determined positive definite function $\varphi$ on $S_{C}$ such that $\varphi\left(s t^{*}\right)=K(s, t)$ for all $s, t \in S_{C}$ ([Ne99, Cor. IV.1.30]).

Since $f$ is non-zero, there exist $v \in \mathcal{H}$ and $s \in S_{C}$ with $f(\rho(s) . v) \neq 0$. Hence $\gamma(s) \neq\{0\}$, i.e., $\gamma\left(S_{C}\right)$ is non-zero, and therefore total because the representation $\rho$ of $S_{C}$ is irreducible. Then [Ne99, Thm. I.1.6] shows that the map $\mathcal{H} \rightarrow \mathbb{C}^{S_{C}}$ defined by $v \mapsto(s \mapsto(f \circ \rho(s))(v))$ induces a surjective isometry $\mathcal{H} \rightarrow \mathcal{H}_{\varphi}$, which then by (4.2) is $S_{C}$-equivariant. In particular $\mathcal{H}_{\varphi}$ is irreducible. On the other hand, since $\gamma$ is constant on the right $\widetilde{H}$-cosets $\widetilde{H} s$, the kernel $K$ on $S_{C} \times S_{C}$ defines a kernel

$$
\bar{K}: \widetilde{H} \backslash S_{C} \times \widetilde{H} \backslash S_{C} \rightarrow \mathbb{C}, \quad(\widetilde{H} s, \widetilde{H} t) \mapsto K(s, t)=\varphi\left(s t^{*}\right) .
$$

Identifying $\widetilde{H} \backslash S_{C}$ with $P_{C}$ via the map

$$
\bar{q}: \widetilde{H} \backslash S_{C} \rightarrow P_{C}, \quad \widetilde{H} s \mapsto q(s)=s^{*} s,
$$

we obtain

$$
\bar{K}: P_{C} \times P_{C} \rightarrow \mathbb{C}, \quad(q(s), q(t)) \mapsto K(s, t)=\varphi\left(s t^{*}\right) .
$$

But the left $\widetilde{H}$-invariance of $K$ together with the existence of an approximate identity in $S_{C}$ also shows that $\varphi$ is left $\widetilde{H}$-invariant as well, so that we find a factorization $\bar{\varphi}: P_{C} \rightarrow \mathbb{C}, q(s) \mapsto \varphi(s)$ which is a positive definite spherical function on $P_{C}$ since the representation of $S_{C}$ on $\mathcal{H}_{\bar{K}}$ is equivalent to the one on $\mathcal{H}_{K}$, hence to the one on $\mathcal{H}$ which is irreducible.

Now we prove the converse and assume that the representation $(\rho, \mathcal{H})$ restricted to $S_{C}$ is equivalent to the natural representation on a space $\left(\pi_{\varphi}, \mathcal{H}_{\varphi}\right)$, where $\varphi$ is a positive definite function on $S_{C}$ which factors through $q$ to a positive definite spherical function $\bar{\varphi}$ on $P_{C}$.

We define a linear functional $f: \mathcal{H}^{0} \rightarrow \mathbb{C}$ by $f(v):=\lim v\left(u_{i}\right)$, where $\left(u_{i}\right)_{i \in I}$ is an approximate identity in $S_{C}$. The limit defining $f$ exists for each $v=\rho(s)$.w, since $v\left(u_{i}\right)=w\left(u_{i} s\right) \rightarrow w(s)$ holds for all $s \in S_{C}$ and $w \in \mathcal{H}_{\varphi}$. Moreover (cf. Theorem 3.1), the equalities

$$
f \circ \pi(h)(\rho(s) \cdot w)=\lim (\rho(h s) \cdot w)\left(u_{i}\right)=\lim w\left(u_{i} h s\right)=w(h s)=w(s)=f(\rho(s) \cdot w)
$$


show that $f$ is $\widetilde{H}$-invariant. In addition, for $s \in S_{C}$ we see that

$$
(f \circ \rho(s))(w)=w(s),
$$

which shows that $f \circ \rho(s)$ is a continuous linear functional on $\mathcal{H}$.

In the classical theory of positive definite spherical functions one requires the $\widetilde{H}$-invariant functional to be a continuous functional on the entire Hilbert space $\mathcal{H}$, so that it can be represented by an $\widetilde{H}$-fixed vector in the Hilbert space. In that setting it is quite easy to show that such a function is unique up to a scalar multiple. We also address the uniqueness question for $f$ if the $S_{C^{-}}$-spherical representation is given, but we only do this for a special case:

We assume that $C$ is hyperbolic (cf. Example 3.7) and proper, i.e., that $C$ does not contain a line. Further we want there to exist a maximal abelian subspace $\mathfrak{a}$ of $\mathfrak{q}$ consisting of hyperbolic elements and a compactly embedded Cartan algebra $\mathfrak{t}^{c}$ of $\mathfrak{g}^{c}$ containing $\mathfrak{a}$. According to $[\mathrm{KrNe} 96]$ such $a$ and $\mathfrak{t}^{c}$ can be found for example if $(\mathfrak{g}, \tau)$ is effective, which means that $\mathfrak{h}$ does not contain a non-trivial ideal of $\mathfrak{g}$.

Proposition 4.4. Let $(\mathfrak{g}, \tau)$ be effective and suppose that $C$ is hyperbolic with $C \cap$ $-C=\{0\}$. Then:

(a) There exists a maximal abelian subspace $\mathfrak{a}$ of $\mathfrak{q}$ consisting of hyperbolic elements.

(b) There exists a compactly embedded Cartan subalgebra $\mathfrak{t}^{c}$ in $\mathfrak{g}^{c}$ containing $\mathfrak{a}$.

Proof. (a) [KrNe96, Thm. VI.6(i)].

(b) [KrNe96, Thm. VI.6(iii), Cor. VII.13].

From now on we assume that Proposition 4.4(a) and (b) hold, but not necessarily that $C \cap-C=\{0\}$. Let $\mathfrak{k}^{c}$ be the uniquely determined maximal compactly embedded subalgebra of $\mathfrak{g}^{c}$ containing $\mathfrak{t}^{c}$. According to [KrNe96, Thm. VIII.1(iii)], there exists a $\mathfrak{k}^{c}$-adapted positive system $\Delta^{+}$such that $\Delta_{\mathfrak{a}}^{+}:=\left.\Delta^{+}\right|_{\mathfrak{a}}$ is a positive system for $\Delta_{\mathfrak{a}}:=\left.\Delta\right|_{\mathfrak{a}} \backslash\{0\}$. We define

$$
\mathfrak{n}:=\sum_{\alpha \in \Delta_{\mathfrak{a}}^{+}} \mathfrak{g}^{\alpha}
$$

and note that $\mathfrak{g}=\mathfrak{h} \oplus \mathfrak{a} \oplus \mathfrak{n}$. Let $A$ and $N$ be the analytic subgroups of $G$ with Lie algebras $\mathfrak{a}$ and $\mathfrak{n}$. Then [KNÓ96, Prop. II.4] shows that the groups $A$, resp. $N$, are closed, simply connected and diffeomorphic to $\mathfrak{a}$, resp. $\mathfrak{n}$, under the exponential mapping. Moreover $A \cap N=\{e\}$, and the map

$$
\Phi: H \times A \times N \rightarrow G, \quad(h, a, n) \mapsto \text { han }
$$

is a diffeomorphism onto its open image. The following result is an immediate consequence of [KNÓ96, Prop. II.6].

Lemma 4.5. $\Gamma(C) \subseteq H A N$.

Lemma 4.6. Let $\left(\pi_{\lambda}, \mathcal{H}\right)$ be an irreducible representation of $G^{c}$ with highest weight $\lambda$ and highest weight vector $v_{\lambda} \in \mathcal{H}$. Suppose that $\pi_{\lambda}$ is related to a continuous representation $\rho$ of $S_{C}$. Then

$$
\operatorname{span}\left(\pi_{\lambda}\left(H^{c}\right) \cdot v_{\lambda}\right)=\operatorname{span}\left(\rho\left(S_{C}\right) \cdot v_{\lambda}\right) .
$$

In particular, $v_{\lambda}$ is a cyclic vector for $H^{c}$. 
Proof. We note first that

$$
\operatorname{span}\left(\pi_{\lambda}\left(H^{c}\right) \cdot v_{\lambda}\right)=\operatorname{span}\left(\widetilde{\pi}_{\lambda}(\widetilde{H}) \cdot v_{\lambda}\right)
$$

in the notation of the Lüscher-Mack Theorem. Further, the diffeomorphism $\Phi$ from (4.3) and Lemma 4.5 induce a real analytic immersion $S_{C} \hookrightarrow \widetilde{H} \times A \times N$. We denote the projection to the middle factor by $a_{\widetilde{H}}: S_{C} \rightarrow A$ and the projection to the left hand factor by $h_{\widetilde{H}}: S_{C} \rightarrow \widetilde{H}$. The Holomorphic Extension Theorem in Appendix $\mathrm{B}$ shows in particular that the map

$$
S_{C} \rightarrow \mathcal{H}, s \mapsto \rho(s) \cdot v_{\lambda}
$$

is real analytic. Now the calculation in the proof of [Ne94c, Prop. III.9] yields

$$
\pi_{\lambda}(s) \cdot v_{\lambda}=a_{H}(s)^{\lambda} \widetilde{\pi}\left(h_{\widetilde{H}}(s)\right) \cdot v_{\lambda}
$$

for all $s \in S_{C}$. This implies the first claim, and the second follows since the representation $\rho$ of $S_{C}$ is irreducible.

Theorem 4.7. Let $\left(\pi_{\lambda}, \mathcal{H}\right)$ be an irreducible representation of $G^{c}$ with highest weight $\lambda$ and suppose that it is $S_{C}$-spherical. So let $\pi$ be related to the continuous representation $\rho$ of $S_{C}$, and let $f: \mathcal{H}^{0} \rightarrow \mathbb{C}$ be an $\widetilde{H}$-invariant functional with $f \circ \rho(s): \mathcal{H} \rightarrow \mathbb{C}$ continuous for all $s \in S_{C}$. Then $f\left(v_{\lambda}\right) \neq 0$, and if $f^{\prime}$ is another $\widetilde{H}$-invariant functional on $\mathcal{H}^{0}$ with the same properties as $f$, then it is a scalar multiple of $f$.

Proof. If $f\left(v_{\lambda}\right)=0$, then $f\left(\widetilde{H} \cdot v_{\lambda}\right)=\{0\}$. Hence Lemma 4.6 shows that $f\left(S_{C} \cdot v_{\lambda}\right)=$ $\{0\}$. Let $s \in S_{C}$. Then it follows that $f \circ \rho(s)$ vanishes on the dense subspace generated by $S_{C} \cdot v_{\lambda}$, whence $f \circ \rho(s)=0$ for all $s \in S_{C}$ and therefore $f=0$. This contradicts the definition of $f$.

Applying the same argument to the functional $f^{\prime}$, we see that the vanishing of $f\left(v_{\lambda}\right) f^{\prime}-f^{\prime}\left(v_{\lambda}\right) f$ on $v_{\lambda}$ implies that this functional on $\operatorname{span}\left(S_{C} \cdot \mathcal{H}\right)$ vanishes, i.e., that $f^{\prime}$ is a scalar multiple of $f$.

Corollary 4.8. Let $\left(\pi_{\lambda}, \mathcal{H}_{\lambda}\right)$ be an $S_{C}$-spherical highest weight representation of $G^{c}$ with highest weight $\lambda$, and $F(\lambda)$ the $K^{c}$-module generated by the highest weight ray, where $K^{c}$ is the analytic subgroup of $G^{c}$ with Lie algebra $\mathfrak{k}^{c}$. Then $F(\lambda)$ is an $\left(H^{c} \cap K^{c}\right)$-spherical representation of $K^{c}$.

Proof. Let $f$ be the $\widetilde{H}$-invariant linear functional on $\operatorname{span}\left(\pi_{\lambda}\left(S_{C}\right) . \mathcal{H}\right)$. Then $\left.f\right|_{F(\lambda)}$ is an $\left(H^{c} \cap K^{c}\right)$-invariant linear functional on $F(\lambda)$ which, in view of Theorem 4.7, is non-zero. Hence it can be represented by an $\left(H^{c} \cap K^{c}\right)$-fixed vector. This means that the irreducible representation $\pi_{\lambda}^{K^{c}}$ of $K^{c}$ on $F(\lambda)$ is $\left(H^{c} \cap K^{c}\right)$-spherical.

Corollary 4.9. If two spherical highest weight representations $\left(\pi_{\varphi_{1}}, \mathcal{H}_{\varphi_{1}}\right)$ and $\left(\pi_{\varphi_{2}}, \mathcal{H}_{\varphi_{2}}\right)$ of $G^{c}$ are equivalent, then $\varphi_{1}$ and $\varphi_{2}$ are multiples of each other. In particular, $\mathcal{H}_{\varphi_{1}}=\mathcal{H}_{\varphi_{2}} \subseteq \mathbb{C}^{S_{C}}$.

Proof. Let $\left(\pi_{\varphi}, \mathcal{H}_{\varphi}\right)$ be any spherical representations of $G^{c}$. In view of Theorem 4.7 it suffices to show that the $\widetilde{H}$-invariant functional $f$ determines the spherical function, i.e., the reproducing kernel, up to a scalar multiple. But Theorem 4.7 together with the proof of Proposition 4.3 show that there exists a constant $c \neq 0$ such that $f(\rho(s) w)=c w(s)=c\left\langle w, K_{s}\right\rangle$ for all $w \in \mathcal{H}_{\varphi}$, proving the claim. 


\section{Positive Definite SPHERICAL FUnCTIONS ON OL'SHANSKIǏ DOMAINS}

Now we come to the classification of the $S_{C}$-spherical unitary representations in the special case where $\mathfrak{g}=\mathfrak{h}_{\mathbb{C}}$ and $\tau$ is the complex conjugation (cf. Def. 1.3). In that case $G=H_{\mathbb{C}}$ is the simply connected group with Lie algebra $\mathfrak{h}_{\mathbb{C}}$ and $H^{c} \cong \Delta_{\widetilde{H}}$, so that $G^{c}=\widetilde{H} \times \widetilde{H}$. Moreover, in this situation $\mathfrak{h}$ admits a compactly embedded Cartan subalgebra $\mathfrak{t}_{\mathfrak{h}}$, and we may assume that $\mathfrak{a}=i \mathfrak{t}_{\mathfrak{h}} \subseteq \mathfrak{q}=i \mathfrak{h}$. We denote the maximal compactly embedded subalgebra of $\mathfrak{h}$ containing $\mathfrak{t}_{\mathfrak{h}}$ by $\mathfrak{k}_{\mathfrak{h}}$. Then we may assume that $\mathfrak{t}^{c}=\mathfrak{t}_{\mathfrak{h}} \oplus \mathfrak{t}_{\mathfrak{h}}$ and $\mathfrak{k}^{c}=\mathfrak{k}_{\mathfrak{h}} \oplus \mathfrak{k}_{\mathfrak{h}}$ (cf. Example 3.7).

The positive domain $P_{C}$ corresponding to $S_{C}$ is the positive domain in the $\mathrm{Ol}^{\prime}$ shanskiü space $H \backslash H_{\mathbb{C}}$ (cf. Remark 2.4). The positive definite spherical functions on the more general positive domains described in Example 1.2 will be treated elsewhere (cf. also Section 7).

Theorem 5.1. The $S_{C}$-spherical representations of $G^{c}=\widetilde{H} \times \widetilde{H}$ are precisely the representations $\pi=\pi_{\lambda} \otimes \pi_{\lambda}^{*}$ on the space $B_{2}\left(\mathcal{H}_{\lambda}\right)$ of Hilbert-Schmidt operators, where $\left(\pi_{\lambda}, \mathcal{H}_{\lambda}\right)$ is a unitary highest weight representation of $\widetilde{H}$ extending to a holomorphic representation of $S_{C}$. The corresponding spherical function $\varphi$ on $S_{C}$ is given by

$$
\varphi(s)=\Theta_{\lambda}\left(s s^{*}\right)
$$

where $\Theta_{\lambda}(s)=\operatorname{tr}\left(\pi_{\lambda}(s)\right)$.

Proof. We have to invoke the parametrization of the irreducible holomorphic representations of $S_{C}$ by their highest weights $\lambda \in i \mathrm{t}^{*}$ (cf. [Ne96, Cor. VI.12]). Then the tensor product $\pi_{\lambda_{1}} \otimes \pi_{\lambda_{2}}^{*}$ is a unitary highest weight representation of $G^{c}=\widetilde{H} \times \widetilde{H}$, and the corresponding irreducible representation of $K^{c}=\widetilde{H}_{K} \times \widetilde{H}_{K}$ is given by $F\left(\lambda_{1}\right) \otimes F\left(\lambda_{2}\right)^{*}$. In Corollary 4.8 we have seen that a necessary condition for

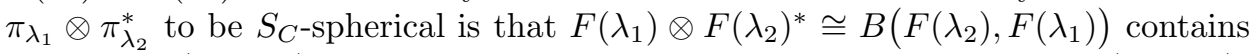
a non-zero $(H \cap K)$-invariant vector. This means exactly that the two $(H \cap K)$ representations $F\left(\lambda_{1}\right)$ and $F\left(\lambda_{2}\right)$ are equivalent, i.e., that $\lambda_{1}=\lambda_{2}$.

Suppose, conversely, that $\lambda_{1}=\lambda_{2}$ and put $\lambda:=\lambda_{1}$. Then the corresponding representation of $S_{W}=S_{C} \times \overline{S_{C}}$ is the representation on the space $B_{2}\left(\mathcal{H}_{\lambda}\right)$ given by $\pi\left(s_{1}, s_{2}\right) \cdot A=\pi_{\lambda}\left(s_{1}\right) A \pi_{\lambda}\left(s_{2}\right)^{*}$, and the restriction to $\Delta_{S_{C}}$ remains irreducible (cf. Proposition 3.8). The subgroup $H=\Delta_{\widetilde{H}} \subseteq G^{c}$ acts by

$$
\pi(h, h) . A=\pi_{\lambda}(h) A \pi_{\lambda}(h)^{-1} .
$$

Therefore $f(A):=\operatorname{tr} A$ defines an $\widetilde{H}$-invariant linear functional on the subspace generated by $\pi_{\lambda}\left(\Delta_{S_{C}}\right) . B_{2}(\mathcal{H}) \subseteq B_{1}(\mathcal{H})$. For $s \in S_{C}$ we have

$$
(f \circ \pi(s, s))(A)=\operatorname{tr}\left(\pi_{\lambda}(s) A \pi_{\lambda}(s)^{*}\right)=\left\langle A, \pi_{\lambda}\left(s^{*} s\right)\right\rangle,
$$

which shows that $f \circ \pi(s, s)$ is continuous. Compare this to $(4.2)$ and set $\gamma(s, s)=$ $\pi_{\lambda}\left(s^{*} s\right)$. Proposition 4.3 and its proof now show that the associated spherical function satisfies $\varphi\left(s t^{*}\right)=\langle\gamma(t, t), \gamma(s, s)\rangle=\operatorname{tr}\left(\pi_{\lambda}\left(t^{*} t s^{*} s\right)\right)=\Theta_{\lambda}\left(s t^{*} t s^{*}\right)$, which implies that $\varphi(s)=\Theta_{\lambda}\left(s s^{*}\right)$.

Theorem 5.1 classifies all positive definite spherical functions of the positive domain $P_{C}$ associated to the semigroup $S_{C}$. In [HiNe96] we studied a family of functions $\varphi_{\mu}: \Gamma\left(C^{0}\right) \rightarrow \mathbb{C}$ with $\mu \in \mathfrak{t}_{h}^{*}+\mathcal{E}_{\mathcal{L}} \subseteq \mathfrak{a}_{\mathbb{C}}^{*}$, where $-i \mathcal{E}_{\mathcal{L}}$ turned out to be the 
interior of the dual cone $C_{\min , \mathfrak{h}}^{\star}$ of the minimal cone in $\mathfrak{t}_{\mathfrak{h}}$ defined by (3.3). These functions were defined by the integrals

$$
\varphi_{\mu}(s)=\int_{H / T_{\mathfrak{h}}} a_{H}(s h)^{\mu-\rho_{\mathfrak{a}}} d \mu_{H / T_{\mathfrak{h}}}\left(h T_{\mathfrak{h}}\right),
$$

where $a_{H}: \Gamma(C) \rightarrow A$ is obtained via Lemma 4.6 as the projection onto the middle factor, $\rho_{\mathfrak{a}}$ is the halfsum of positive roots in $\Delta_{\mathfrak{a}}^{+}, T_{\mathfrak{h}}$ is the analytic subgroup of $H$ with Lie algebra $\mathfrak{t}_{\mathfrak{h}}$, and $\mu_{H / T_{\mathfrak{h}}}$ is a suitable $H$-invariant measure. If we lift these functions to $S_{C}=\widetilde{\Gamma}\left(C^{0}\right)$, the resulting function $\widetilde{\varphi}_{\mu}$ is given by an integral of the form

$$
\widetilde{\varphi}_{\mu}(s)=\int_{\widetilde{H} / \widetilde{T}_{\mathfrak{h}}} a_{\widetilde{H}}(s h)^{\mu-\rho_{\mathfrak{a}}} d \mu_{\widetilde{H} / \widetilde{T}_{\mathfrak{h}}}\left(h \widetilde{T}_{\mathfrak{h}}\right),
$$

since the center of $\widetilde{H}$ is contained in the analytic subgroup $\widetilde{T}_{\mathfrak{h}}$ of $\widetilde{H}$ with Lie algebra $\mathfrak{t}_{\mathfrak{h}}$, so that we can identify $H / T_{\mathfrak{h}}$ with $\widetilde{H} / \widetilde{T}_{\mathfrak{h}}$. In the following theorem we determine those $\widetilde{\varphi}_{\mu}$ which are positive definite spherical functions on $S_{C}$ in the sense of Definition 2.2.

Theorem 5.2. The function $\widetilde{\varphi}_{\mu}: S_{C} \rightarrow \mathbb{C}$ is a positive definite spherical function if and only if $\lambda:=\frac{1}{2}\left(\mu-\rho_{\mathfrak{a}}\right)$ is dominant integral with respect to the positive system $\Delta_{\mathfrak{h}, k}^{+}$of $\Delta\left(\mathfrak{k}_{\mathfrak{h}}, \mathfrak{t}_{\mathfrak{h}}\right)$ inherited from $\Delta_{\mathfrak{a}}^{+}$.

Proof. Suppose first that $\lambda$ is dominant integral with respect to $\Delta_{\mathfrak{h}, k}^{+}$. Then the fact that $\lambda+\frac{\rho_{\mathfrak{a}}}{2}=\frac{\mu}{2} \in i \operatorname{int}\left(C_{\min , \mathfrak{h}}^{\star}\right)$ implies that we have a corresponding unitary highest weight representation $\left(\pi_{\lambda}, \mathcal{H}_{\lambda}\right)$ of $\widetilde{H}$ which is square integrable modulo the center (cf. [Ne95b, Thm. III.15]). Hence, using (4.4) in the appropriate setting, we can calculate

$$
\begin{aligned}
\widetilde{\varphi}_{\mu}(s) & =\int_{\widetilde{H} / \widetilde{T}_{\mathfrak{h}}} a_{\widetilde{H}}(s h)^{2 \lambda} d \mu_{\widetilde{H} / \widetilde{T}_{\mathfrak{h}}}\left(h \widetilde{T}_{\mathfrak{h}}\right) \\
& =\int_{\widetilde{H} / \widetilde{T}_{\mathfrak{h}}}\left\|\pi_{\lambda}(s h) \cdot v_{\lambda}\right\|^{2} d \mu_{\widetilde{H} / \widetilde{T}_{\mathfrak{h}}}\left(h \widetilde{T}_{\mathfrak{h}}\right) \\
& =\int_{\widetilde{H} / \widetilde{T}_{\mathfrak{h}}}\left\langle\pi_{\lambda}\left(s^{*} s\right) \pi_{\lambda}(h) \cdot v_{\lambda}, \pi_{\lambda}(h) \cdot v_{\lambda}\right\rangle d \mu_{\widetilde{H} / \widetilde{T}_{\mathfrak{h}}}\left(h \widetilde{T}_{\mathfrak{h}}\right) \\
& =\frac{1}{d\left(\pi_{\lambda}\right)} \operatorname{tr} \pi_{\lambda}\left(s^{*} s\right) .
\end{aligned}
$$

According to the hyperbolicity of the cone $C$, we have $C \cap i \mathfrak{t}_{\mathfrak{h}} \subseteq\left(\Delta_{p}^{+}\right)^{\star}$, so that, in view of [Ne96, Cor. IV.12], $\pi_{\lambda}$ extends to a holomorphic representation of $S_{C}$. Thus $\widetilde{\varphi}_{\mu}: S_{C} \rightarrow \mathbb{C}$ is one of the positive definite spherical functions provided by Theorem 5.1.

Suppose conversely that the function $\widetilde{\varphi}_{\mu}$ is positive definite spherical. Then the classification of positive definite spherical functions shows that there exists a unitary highest weight representation $\left(\pi_{\lambda}, \mathcal{H}_{\lambda}\right)$ of $\widetilde{H}$ such that

$$
\varphi_{\mu}(s)=C \operatorname{tr} \pi_{\lambda}\left(s^{*} s\right)
$$

holds for all $s \in S$ and $C>0$. To see which relation we have between $\lambda$ and $\mu$ we have to calculate the asymptotics of the function on the right. So let $\mathcal{P}$ denote the set of all $\left(\mathfrak{t}_{\mathfrak{h}}\right)_{\mathbb{C}}$ weights on the space $\mathcal{H}_{\lambda}^{K_{\mathfrak{h}}}$ of $K_{\mathfrak{h}}$-finite vectors in $\mathcal{H}$, which, as 


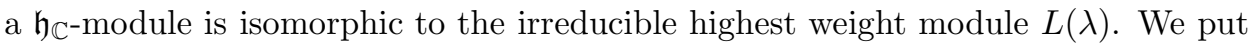
$\psi(s):=\operatorname{tr} \pi_{\lambda}\left(s^{*} s\right)$ for $s \in S$. Then for $a \in A \cap S_{C}$ we have

$$
\psi(a)=\operatorname{tr} \pi_{\lambda}\left(a^{2}\right)=\sum_{\alpha \in \mathcal{P}} m_{\alpha} a^{2 \alpha} .
$$

Moreover,

$$
\psi(a) a^{-2 \lambda}=\sum_{\alpha \in \mathcal{P}} m_{\alpha} a^{2(\alpha-\lambda)} .
$$

Now Lebesgue's Dominated Convergence Theorem and $m_{\lambda}=1$ imply for each $X$ in the cone $\operatorname{int}\left(\Delta\left(\mathfrak{k}_{\mathfrak{h}}, \mathfrak{t}_{\mathfrak{h}}\right)^{+}\right)^{\star} \subseteq i \mathfrak{t}_{\mathfrak{h}}=\mathfrak{a}$ that

$$
\lim _{t \rightarrow \infty} \psi(\exp (t X))(\exp (t X))^{-2 \lambda}=1 .
$$

On the other hand we know from [HiNe96, Thm. II.2.19] that

$$
\lim _{t \rightarrow \infty} \varphi_{\mu}(\exp (t X))(\exp (t X))^{\rho_{\mathfrak{a}}-\mu}=c_{1} c_{\mathcal{M}}(\mu),
$$

where $c_{\mathcal{M}}$ is the $c$-function for the space $\mathcal{M}=H \backslash H_{\mathbb{C}}$ and $c_{1}$ is a suitable constant. It follows in particular that the limit on the right hand side is independent of $X$. Hence we obtain

$$
\lim _{t \rightarrow \infty}(\exp (t X))^{\rho_{\mathfrak{a}}-\mu+2 \lambda}=c_{1} c_{\mathcal{M}}(\lambda) .
$$

From that we conclude that $\rho_{\mathfrak{a}}-\mu+2 \lambda=0$. It follows in particular that $\mu \in \mathfrak{a}^{*}=i \mathfrak{t}_{\mathfrak{h}}^{*}$ and that $\lambda=\frac{1}{2}\left(\mu-\rho_{\mathfrak{a}}\right)$ is dominant integral with respect to $\Delta_{\mathfrak{h}, k}^{+}$. This completes the proof.

Example 5.3. We illustrate Theorem 5.2 by the example where $H$ is compact. Here $H \backslash H_{\mathbb{C}}$ is a Riemannian symmetric space, the integral formula for $\widetilde{\varphi}_{\mu}$ is just Harish-Chandra's integral formula (cf. [Hel84, p. 418]), and writing the spherical function using the character of a representation of $H$, in view of Weyl's character formula, yields Harish-Chandra's spherical function expansion in the complex case ([Hel84, Ch. IV, Thm. 5.7]).

Remark 5.4. We have already seen in Theorem 5.2 that for an integral spherical function $\varphi_{\mu}$ to be positive definite the parameter $\mu$ has to satisfy an additional integrality condition. On the other hand one could ask which positive definite functions $\bar{\varphi}$ on $P_{C}$ are spherical in the sense of [HiNe96, Def. II.2.1], i.e., satisfy

$$
\int_{\widetilde{H} / \widetilde{T}_{\mathfrak{h}}} \bar{\varphi}(q(a h b)) d \mu_{\widetilde{H} / \widetilde{T}_{\mathfrak{h}}}\left(h \widetilde{T}_{\mathfrak{h}}\right)=\bar{\varphi}(q(a)) \bar{\varphi}(q(b))
$$

for $a, b \in S_{C} \cap \operatorname{Exp}\left(i \mathfrak{t}_{\mathfrak{h}}\right)$. Theorem 5.1 says that each $\bar{\varphi}$ is of the form

$$
\bar{\varphi}(q(s))=\varphi(s)=c \Theta_{\lambda}\left(s s^{*}\right)=c \operatorname{tr}\left(\pi_{\lambda}\left(s s^{*}\right)\right),
$$

where $\Theta_{\lambda}$ is a suitable highest weight character on $S_{C}$ and $c>0$ is a constant.

Suppose that the above integrals exist, and put $s:=a=b$. Then

$$
\bar{\varphi}(q(s h s))=c \operatorname{tr}\left(\pi_{\lambda}\left(s h s^{*} s^{*} h^{-1} s\right)\right)=c \operatorname{tr}\left(\pi_{\lambda}\left(h s^{2} h^{-1} s^{2}\right)\right) .
$$

Since the highest weight vector $v_{\lambda}$ is an eigenvector for $\pi_{\lambda}(s)$, the trace class operator $\pi_{\lambda}\left(s^{2}\right)$ can be written as $\pi_{\lambda}\left(s^{2}\right)=\sum_{n=1}^{\infty} P_{v_{n}}$, where $P_{v_{n}}(x)=\left\langle x, v_{n}\right\rangle v_{n}$, and 
$\left(v_{n}\right)_{n \in \mathbb{N}}$ is an orthogonal sequence of eigenvectors of $\pi_{\lambda}\left(s^{2}\right)$, where $v_{1}=d v_{\lambda}$. Hence

$$
\begin{aligned}
\operatorname{tr}\left(\pi_{\lambda}\left(h s^{2} h^{-1} s^{2}\right)\right) & \geq d^{2} \operatorname{tr}\left(\pi_{\lambda}\left(h s^{2} h^{-1}\right) P_{v_{\lambda}}\right) \\
& \geq d^{4} \operatorname{tr}\left(\pi_{\lambda}(h) P_{v_{\lambda}} \pi_{\lambda}\left(h^{-1}\right) P_{v_{\lambda}}\right) \\
& \geq d^{4} \operatorname{tr}\left(P_{\pi_{\lambda}(h) \cdot v_{\lambda}} P_{v_{\lambda}}\right)=d^{4}\left|\left\langle\pi_{\lambda}(h) \cdot v_{\lambda}, v_{\lambda}\right\rangle\right|^{2} .
\end{aligned}
$$

Thus the existence of the integral above implies that the highest weight representation $\left(\pi_{\lambda}, \mathcal{H}\right)$ of $\widetilde{H}$ is square integrable in the sense of [Ne95b, Def. III.4]. Then $\mu:=2 \lambda+\rho_{\mathfrak{a}} \in i \operatorname{int}\left(C_{\min , \mathfrak{h}}^{\star}\right)$ and, as we have seen in the proof of Theorem 5.2,

$$
\widetilde{\varphi}_{\mu}(s)=\frac{1}{d\left(\pi_{\lambda}\right)} \operatorname{tr} \pi_{\lambda}\left(s s^{*}\right) .
$$

We have already seen in [HiNe96, Thm. II.2.6] that the functions $\widetilde{\varphi}_{\mu}$ satisfy the above integral equation, which implies in particular that $c=\frac{1}{d\left(\pi_{\lambda}\right)}$ and $\varphi=\widetilde{\varphi}_{\mu}$.

In this setting the integral formula for the spherical functions has an interesting interpretation. For $h \in \widetilde{H}$ and $A \in B(\mathcal{H})$ we put $h . A:=\pi_{\lambda}(h) A \pi_{\lambda}\left(h^{-1}\right)$. Let $P_{x, y}$ and $P_{v, w}$ denote the rank one operators on $\mathcal{H}_{\lambda}$ defined by $P_{x, y}(u)=\langle u, y\rangle x$ and $P_{v, w}(u)=\langle u, w\rangle v$. For $H_{1}:=(\widetilde{H}, \widetilde{H})$ and $Z:=Z(\widetilde{H}) \cap H_{1}$ we write $\mu:=\mu_{H_{1} / Z}$ for the invariant measure on $H_{1} / Z$. Then the orthogonality relations for square integrable highest weight representations imply that

$$
\begin{aligned}
\int_{H_{1} / Z} \operatorname{tr}\left(h \cdot P_{x, y} P_{v, w}\right) d \mu(h Z) & =\int_{H_{1} / Z} \operatorname{tr}\left(P_{h . x, h . y} P_{v, w}\right) d \mu(h Z) \\
& =\int_{H_{1} / Z}\langle v, h . y\rangle\langle h . x, w\rangle d \mu(h Z) \\
& =\frac{1}{d\left(\pi_{\lambda}\right)}\langle v, w\rangle \cdot\langle x, y\rangle \\
& =\frac{1}{d\left(\pi_{\lambda}\right)} \operatorname{tr}\left(P_{v, w}\right) \operatorname{tr}\left(P_{x, y}\right)
\end{aligned}
$$

Next the Monotone Convergence Theorem implies that the integral

$$
\int_{H_{1} / Z} \operatorname{tr}(h \cdot A B) d \mu(h Z)
$$

exists and equals

$$
\frac{1}{d\left(\pi_{\lambda}\right)} \operatorname{tr}(A) \operatorname{tr}(B)
$$

whenever $A$ and $B$ are positive trace class operators. Using the fact that each trace class operator is a linear combination of positive ones, we see that

$$
\int_{H_{1} / Z} \operatorname{tr}(h . A B) d \mu(h Z)=\frac{1}{d\left(\pi_{\lambda}\right)} \operatorname{tr}(A) \operatorname{tr}(B)
$$


for all $A, B \in B_{1}(\mathcal{H})$. For $s, t \in S_{C}$ we obtain in particular

$$
\begin{aligned}
\int_{\widetilde{H}_{1} / \widetilde{T}_{\mathfrak{h}}} \bar{\varphi}(q(s h t)) d \mu\left(h \widetilde{T}_{\mathfrak{h}}\right) & =\int_{H_{1} / Z} \bar{\varphi}(q(s h t)) d \mu(h Z) \\
& =\frac{1}{d\left(\pi_{\lambda}\right)} \int_{H_{1} / Z} \operatorname{tr}\left(\pi_{\lambda}\left(s h t t^{*} h^{-1} s^{*}\right)\right) d \mu(h Z) \\
& =\frac{1}{d\left(\pi_{\lambda}\right)} \int_{H_{1} / Z} \operatorname{tr}\left(\pi_{\lambda}\left(h t t^{*} h^{-1} s^{*} s\right)\right) d \mu(h Z) \\
& =\frac{1}{d\left(\pi_{\lambda}\right)} \int_{H_{1} / Z} \operatorname{tr}\left(h . \pi_{\lambda}\left(t t^{*}\right) \pi_{\lambda}\left(s^{*} s\right)\right) d \mu(h Z) \\
& =\frac{1}{d\left(\pi_{\lambda}\right)^{2}} \operatorname{tr}\left(\pi_{\lambda}\left(t t^{*}\right)\right) \operatorname{tr}\left(\pi_{\lambda}\left(s^{*} s\right)\right) \\
& =\bar{\varphi}(q(s)) \bar{\varphi}(q(t)) .
\end{aligned}
$$

Thus we have obtained the integral equation for the spherical function from a more general integral identity for trace class operators which in turns follows from the orthogonality relations for square integrable representations.

\section{Holomorphic Positive Definite Spherical Functions}

Let $\left(S_{C},{ }^{*}\right)$ and $S_{W}$ be as in Example 3.7. Then $S_{C}$ can be viewed as the positive domain associated to $\left(S_{W},{ }^{*}, \tau\right)$, where $(s, t)^{*}=\left(s^{*}, t^{*}\right)$ and $\tau(s, t)=(t, s)$ (cf. Example 1.4). The canonical map

$$
q: S_{W} \rightarrow S_{C}, \quad(s, t) \mapsto t^{*} s
$$

(cf. (1.1)) is holomorphic, and any kernel on $S_{C}$ associated to a holomorphic function $\varphi: S_{C} \rightarrow \mathbb{C}$ via (2.1) is holomorphic in both arguments.

A continuous representation $(\rho, \mathcal{H})$ of $S_{C}$ by bounded operators is called holomorphic if the map $\rho: S_{C} \rightarrow B(\mathcal{H})$ is holomorphic as a Banach space valued map (cf. [Ne95a] and [HiNe93, Chap. 9])). According to [Ne94b, Thm. III.8] (cf. also [Ne96, Th. IV.3]) the irreducible holomorphic representations act by trace class operators and hence admit characters (cf. Example 2.6). The functions on $S_{C}$ that occur in this way are called the holomorphic characters of $S_{C}$.

Proposition 6.1. Let $\varphi: S_{C} \rightarrow \mathbb{C}$ be a holomorphic positive definite spherical function. Then the representation $\left(\pi_{\varphi}, \mathcal{H}_{\varphi}\right)$ of $S_{W}$, given by

$$
\left(\pi_{\varphi}(s, t) . f\right)(x)=f\left(t^{*} x s\right) \quad \text { for } \quad f \in \mathcal{H}_{\varphi}, x \in S_{C},
$$

is holomorphic. Moreover it is the holomorphic extension of a representation $\left(\pi_{1} \otimes \pi_{1}^{*}, \mathcal{H}_{1} \widehat{\otimes} \mathcal{H}_{1}^{*}\right)$ of $\widetilde{H} \times \widetilde{H}$, where $\left(\pi_{1}, \mathcal{H}_{1}\right)$ is a highest weight representation of $\widetilde{H}$.

Proof. In view of [HiNe93, Lemma 9.7], for the first claim it suffices to show that the functions

$$
S_{W} \rightarrow \mathbb{C}, s \mapsto\left\langle\pi_{\varphi}(s, t) \cdot K_{z}, K_{w}\right\rangle
$$

are holomorphic for all $z, w \in S_{C}$, where $K_{z}(x)=K(x, z)=\varphi\left(x z^{*}\right)$. But this follows from

$$
\left\langle\pi_{\varphi}(s, t) \cdot K_{z}, K_{w}\right\rangle=\left\langle K_{t z s^{*}}, K_{w}\right\rangle=K\left(w, t z s^{*}\right)=K\left(t^{*} w s, z\right) .
$$


It follows from [Ne96, Cor. IV.12] that the representation $\left(\pi_{\varphi}, \mathcal{H}_{\varphi}\right)$ is the holomorphic extension of an irreducible unitary highest weight representation of $\widetilde{H} \times \widetilde{H}$. The choice of the complex structure then forces this representation to be of the form $\left(\pi_{1} \otimes \pi_{2}^{*}, \mathcal{H}_{1} \widehat{\otimes} \mathcal{H}_{2}\right)$ with highest weight representations $\left(\pi_{i}, \mathcal{H}_{i}\right)$ of $\widetilde{H}$ for $i=1,2$ (cf. Example 3.7). The representation space $\mathcal{H}_{\varphi}$ is invariant under the antilinear involution defined by

$$
\sigma(f)(s):=f^{*}(s):=\overline{f\left(s^{*}\right)}
$$

satisfying

$$
\left(\left(\sigma \circ \rho_{t}\right) . f\right)(s)=\overline{f\left(s^{*} t\right)}=\overline{f\left(\left(t^{*} s\right)^{*}\right)}=f^{*}\left(t^{*} s\right)=\left(\left(\lambda_{t} \circ \sigma\right) . f\right)(s),
$$

where $\left(\rho_{s} . f\right)(t)=f(t s)$ and $\left(\lambda_{s} . f\right)(t)=f\left(s^{*} t\right)$. This shows that

$$
\sigma \circ \pi_{\varphi}(s, t)=\sigma \circ \rho_{s} \circ \lambda_{t}=\lambda_{s} \circ \rho_{t} \circ \sigma=\pi_{\varphi}(t, s) \circ \sigma .
$$

For any Hilbert space $\mathcal{H}$ consider the antilinear isomorphism

$$
\iota: \mathcal{H} \rightarrow \mathcal{H}^{*}, v \mapsto(w \mapsto\langle w, v\rangle)
$$

In the case of $\mathcal{H}_{\varphi}$ one easily checks that

$$
\iota \circ \pi_{\varphi}(s, t)=\pi_{\varphi}^{*}(s, t) \circ \iota .
$$

But then (6.1) and (6.2) imply that the Hilbert space isomorphism $\iota \circ \sigma$ intertwines $\pi_{\varphi}^{*}$ and $\pi_{\varphi} \circ \tau$, where $\tau(s, t)=(t, s)$ (cf. Example 1.4). But $\pi_{\varphi} \circ \tau \cong \pi_{\varphi}^{*}$ implies

$$
\pi_{1}^{*} \otimes \pi_{2} \cong \pi_{\varphi}^{*} \cong \pi_{\varphi} \circ \tau \cong \pi_{2}^{*} \otimes \pi_{1},
$$

so that $\pi_{1} \cong \pi_{2}$.

Note that the representation $\left(\pi_{1} \otimes \pi_{1}^{*}, \mathcal{H}_{1} \widehat{\otimes} \mathcal{H}_{1}^{*}\right)$ of $\widetilde{H} \times \widetilde{H}$ and $S_{W}$ is canonically equivalent to the representation of $\widetilde{H} \times \widetilde{H}$, resp. $S_{W}$, on the Hilbert-Schmidt operators $B_{2}\left(\mathcal{H}_{1}\right)$ defined by

$$
(s, t) \cdot A=\pi_{1}(s) \circ A \circ \pi_{1}\left(t^{*}\right) .
$$

Theorem 6.2. The holomorphic positive definite spherical functions on $S_{C}$ are the multiples of the irreducible holomorphic characters.

Proof. In Example 2.6 we have seen that each irreducible holomorphic character is a holomorphic positive definite spherical function on $S_{C}$. So it remains to be shown that each holomorphic positive definite spherical function is such a character.

Let $\varphi$ be an exponentially bounded positive definite spherical function on $S_{C}$ and $\pi_{\varphi} \cong \pi_{1} \otimes \pi_{1}^{*}$ the corresponding irreducible holomorphic representation of $S_{W}$ on $\mathcal{H}_{\varphi} \subseteq \operatorname{Hol}\left(S_{C}\right)$ (Proposition 6.1). Let $\Theta: S_{C} \rightarrow \mathbb{C}$ be the character of the holomorphic representation $\left(\pi_{1}, \mathcal{H}_{1}\right)$. Then $\Theta$ is a holomorphic positive definite spherical function. We want to show that $\varphi$ is a multiple of $\Theta$. Consider the map $\gamma: S_{W} \rightarrow B_{2}\left(\mathcal{H}_{1}\right),(s, t) \mapsto \pi_{1}\left(s^{*} t\right)$ and set

$$
\begin{aligned}
K_{\gamma}\left(\left(s_{1}, s_{2}\right),\left(t_{1}, t_{2}\right)\right) & =\left\langle\gamma\left(t_{1}, t_{2}\right), \gamma\left(s_{1}, s_{2}\right)\right\rangle=\operatorname{tr}\left(\gamma\left(t_{1}, t_{2}\right) \gamma\left(s_{1}, s_{2}\right)^{*}\right) \\
& =\Theta\left(t_{1}^{*} t_{2} s_{2}^{*} s_{1}\right)=\Theta\left(t_{2} s_{2}^{*} s_{1} t_{1}^{*}\right) \\
& =\Theta\left(q\left(s_{1} t_{1}^{*}, s_{2} t_{2}^{*}\right)\right)=\Theta\left(q\left(\left(s_{1}, s_{2}\right)\left(t_{1}, t_{2}\right)^{*}\right)\right) .
\end{aligned}
$$

Then [Ne99, Thm. I.1.6] shows that the map $\Phi: B_{2}\left(\mathcal{H}_{1}\right) \rightarrow \mathcal{H}_{K_{\gamma}}$ defined by

$$
\Phi(A)(s, t)=\left\langle A, \pi_{1}\left(s^{*} t\right)\right\rangle=\operatorname{tr}\left(A \pi_{1}\left(t^{*}\right) \pi_{1}(s)\right)=\operatorname{tr}\left(\pi_{1}(s) A \pi_{1}\left(t^{*}\right)\right)
$$


is a Hilbert space isomorphism, since $\pi_{1} \otimes \pi_{1}^{*}$ is an irreducible representation of $S_{W}$ and it is also $S_{W}$-equivariant. On the other hand, $K_{\gamma}$ factors through $q: S_{W} \rightarrow S_{C}$ to the kernel $K_{\Theta}(q(s), q(t))=\Theta\left(q\left(s t^{*}\right)\right)$ associated to $\Theta$ via (2.1). This means that $\mathcal{H}_{K_{\gamma}}$ can be identified with $\mathcal{H}_{\Theta}$ as an $S_{W}$-representation. Thus we have the following sequence of equivalent $S_{W}$, and hence $G^{c}$-representations:

$$
\mathcal{H}_{\varphi} \cong B_{2}\left(\mathcal{H}_{1}\right) \cong \mathcal{H}_{K_{\gamma}} \cong \mathcal{H}_{\Theta}
$$

Now Corollary 4.9 shows that $\varphi$ is a multiple of $\Theta$.

Theorem 5.1 and its proof yield:

Proposition 6.3. The holomorphic spherical representations of $S_{W}$ correspond to the representations $\pi=\pi_{\lambda} \otimes \pi_{\lambda}^{*}$ of $G^{c}=\widetilde{H} \times \widetilde{H}$ on the space $B_{2}\left(\mathcal{H}_{\lambda}\right)$ of HilbertSchmidt operators, where $\left(\pi_{\lambda}, \mathcal{H}_{\lambda}\right)$ is a unitary highest weight representation extending to a holomorphic representation of $S_{C}$. The corresponding holomorphic spherical function $\widehat{\varphi}$ on $S_{W}$ is given by

$$
\widehat{\varphi}\left(s_{1}, s_{2}\right)=\operatorname{tr} \pi_{\lambda}\left(s_{1} s_{2}^{*}\right) .
$$

Proof. All we have to note is that the holomorphic continuation of the spherical function $\varphi(s)=\Theta_{\lambda}\left(s s^{*}\right)$ to a holomorphic spherical function on $S_{W}$ given by $\widehat{\varphi}\left(s_{1}, s_{2}\right)=\Theta_{\lambda}\left(s_{1} s_{2}^{*}\right)$ is, by the uniqueness of holomorphic continuation, unique.

Example 6.4. We illustrate Theorem 6.2 and Proposition 6.3 in the case that $H$ is compact and $C=i \mathfrak{h}$. Then $S_{C}=H_{\mathbb{C}}$ and $S_{W}=H_{\mathbb{C}} \times \overline{H_{\mathbb{C}}}$. The irreducible holomorphic representations of $H_{\mathbb{C}}$ are complex finite dimensional representations of highest weight. Thus the holomorphic positive definite spherical functions of $H_{\mathbb{C}}$ are, up to scalar multiples, nothing but the group characters, and the resulting holomorphic representations of $H_{\mathbb{C}} \times \overline{H_{\mathbb{C}}}$ are the representations of the form $\pi \otimes \pi^{*}$, where $\pi^{*}=\overline{\widehat{\pi}}$ is the complex conjugate of the contragredient representation.

Right multiplication of $\widetilde{H}$ on $S_{C}$ yields for each $X \in \mathfrak{h}$ a holomorphic vector field $r(X)$ on $S_{C}$ defined by

$$
r(X)(s):=\left.\frac{d}{d t}\right|_{t=0} s \cdot \operatorname{Exp}(t X)
$$

Let $B$ be a Banach space and $\operatorname{Hol}\left(S_{C}, B\right)$ the space of holomorphic $B$-valued maps. Then to each element $D \in \mathcal{U}\left(\mathfrak{h}_{\mathbb{C}}\right)$ there corresponds a holomorphic differential operator $r(D)$ on $\operatorname{Hol}\left(S_{C}, B\right)$ defined by

$$
(r(X) . f)(s)=\left.\frac{d}{d t}\right|_{t=0} f(s \operatorname{Exp} t X)
$$

for $X \in \mathfrak{h}_{\mathbb{C}}$ and its extension to $\mathcal{U}\left(\mathfrak{h}_{\mathbb{C}}\right)$. In this sense the center $\mathcal{Z}:=Z\left(\mathcal{U}\left(\mathfrak{h}_{\mathbb{C}}\right)\right)$ acts on the space $\operatorname{Hol}\left(S_{C}\right)$ and corresponds to the space $\mathbb{D}\left(S_{C}\right)$ of $(\widetilde{H} \times \widetilde{H})$-invariant holomorphic differential operators on the positive domain $S_{C}$.

Let $\chi \in \widehat{\mathcal{Z}}$ be a central character, i.e., a multiplicative functional on $\mathcal{Z}$. Then the corresponding eigenspace $\operatorname{Hol}\left(S_{C}\right)_{\chi}$ is invariant under $\widetilde{H} \times \widetilde{H}$, hence also invariant with respect to the action of $S_{W}=S_{C} \times \overline{S_{C}}$ given by $\left(\rho\left(s_{1}, s_{2}\right) . f\right)(s)=f\left(s_{2}^{*} s s_{1}\right)$, i.e., the action induced by the natural right action on $S_{C}$.

In Theorem 6.2 we have seen that each holomorphic spherical function is an irreducible holomorphic character $\Theta_{\pi_{\lambda}}$, where $\left(\pi_{\lambda}, \mathcal{H}_{\lambda}\right)$ is an irreducible representation of $S_{C}$ extending the representation of $\widetilde{H}$ with highest weight $\lambda$. Let $\chi_{\lambda} \in \widehat{\mathcal{Z}}$ denote 
the central character corresponding to the representation of $\mathcal{U}\left(\mathfrak{h}_{\mathbb{C}}\right)$ with highest weight $\lambda$. We claim that $\mathcal{H}_{\Theta_{\pi_{\lambda}}} \subseteq \operatorname{Hol}\left(S_{C}\right)_{\chi_{\lambda}}$.

For the proof we will have to calculate the action of the enveloping algebra on the character $\Theta:=\Theta_{\pi_{\lambda}}$. The most convenient way to do this is to use the following lemma.

Lemma 6.5. Let $(\pi, \mathcal{H})$ be an irreducible holomorphic representation of $S_{C}$ such that $\pi\left(S_{C}\right) \subseteq B_{1}(\mathcal{H})$. Then the following assertions hold:

(i) For each $D \in \mathcal{U}\left(\mathfrak{h}_{\mathbb{C}}\right)$ and $s \in S_{C}$ the operator $d \pi(D) \pi(s)$ is bounded and its adjoint is given by $\pi\left(s^{*}\right) d \pi\left(D^{*}\right)$, where $D \mapsto D^{*}$ is the antilinear antiautomorphism extending the map $X \mapsto-\bar{X}, \mathfrak{h}_{\mathbb{C}} \rightarrow \mathfrak{h}_{\mathbb{C}}$.

(ii) $\pi: S_{C} \rightarrow B_{1}(\mathcal{H})$ is a holomorphic Banach space valued map and satisfies

$$
(r(D) . \pi)(s)=\pi(s) d \pi(D)
$$

for all $D \in \mathcal{U}\left(\mathfrak{h}_{\mathbb{C}}\right)$.

(iii) $r(D) . \pi=\chi_{\lambda}(D) \pi$ holds for all $D \in \mathcal{Z}$.

Proof. (i) Since $\pi$ is holomorphic, we have $\pi(s) . \mathcal{H} \subseteq \mathcal{H}^{\infty}$, and hence the operator $d \pi(D) \pi(s)$ is defined on $\mathcal{H}$. On the other hand, for $v, w \in \mathcal{H}^{\infty}$, we have

$$
\langle d \pi(D) \pi(s) \cdot v, w\rangle=\left\langle\pi(s) \cdot v, d \pi\left(D^{*}\right) \cdot w\right\rangle=\left\langle v, \pi\left(s^{*}\right) d \pi\left(D^{*}\right) \cdot w\right\rangle,
$$

where $D \mapsto D^{*}$ is the extension of the antiautomorphism $X \mapsto-X$ of $\mathfrak{h}_{\mathbb{C}}$ to $\mathcal{U}\left(\mathfrak{h}_{\mathbb{C}}\right)$. Hence the adjoint operator $(d \pi(D) \pi(s))^{*}$ has a dense domain containing $\mathcal{H}^{\infty}$. By the Closed Graph Theorem we conclude that the operator $d \pi(D) \pi(s)$, which is closable and everywhere defined, is bounded. From this it also follows that $\pi\left(s^{*}\right) d \pi\left(D^{*}\right)$ is bounded and that it coincides with the adjoint of $d \pi(D) \pi(s)$. This proves (i).

(ii) First we show that $\pi(s) d \pi(D)$ is a trace class operator for all $s \in S_{C}$ and $D \in \mathcal{U}\left(\mathfrak{h}_{\mathbb{C}}\right)$. We write $s=s_{1} s_{2}$ with $s_{1}, s_{2} \in S_{C}$ (cf. [HiNe93, Prop. 3.19]). Then (i) implies that

$$
\pi(s) d \pi(D)=\pi\left(s_{1}\right)\left(\pi\left(s_{2}\right) d \pi(D)\right) \in B_{1}(\mathcal{H}) B(\mathcal{H}) \subseteq B_{1}(\mathcal{H}) .
$$

To see that the map $\pi: S_{C} \rightarrow B_{1}(\mathcal{H})$ is holomorphic, we first note that it is locally bounded in the norm $\|\cdot\|_{1}$ of $B_{1}(\mathcal{H})$. In fact, let $s \in S_{C}$ and write it as $s=s_{1} s_{2}$. Pick a compact neighborhood $U$ of $s_{2}$ in $S_{C}$ and note that the holomorphy of $\pi$ as a $B(\mathcal{H})$-valued map shows that we can find a constant $C>0$ such that $\|\pi(u)\| \leq C$ for all $u \in U$. Then $s_{1} U$ is a neighborhood of $s$, and

$$
\left\|\pi\left(s_{1} t\right)\right\|_{1}=\left\|\pi\left(s_{1}\right)\right\|_{1} \cdot\|\pi(t)\| \leq C\left\|\pi\left(s_{1}\right)\right\|_{1}
$$

holds for all $t \in U$. It now suffices to show that for $P_{v, w}(x)=\langle x, w\rangle v$ the function $s \mapsto \operatorname{tr}\left(\pi(s) P_{v, w}\right), S_{C} \rightarrow \mathbb{C}$ is holomorphic for all $v, w \in \mathcal{H}$. Then [HiNe93, Lemma 9.7(ii)] shows that $\pi$ is real analytic, and since [HiNe93, Lemma 9.7(i)] implies that it is partially holomorphic, it must be holomorphic. But for $v, w \in \mathcal{H}$ we have

$$
\operatorname{tr}\left(\pi(s) P_{v, w}\right)=\operatorname{tr} P_{\pi(s) \cdot v, w}=\langle\pi(s) . v, w\rangle,
$$

which is trivially holomorphic in $s$. Thus $\pi: S_{C} \rightarrow B_{1}(\mathcal{H})$ is a holomorphic map.

Next we show that $r(X) \cdot \pi=\pi \cdot d \pi(X)$ holds for all $X \in \mathfrak{h}$. Recall first that the pairing

$$
B_{1}(\mathcal{H}) \times B(\mathcal{H}) \rightarrow \mathbb{C},(A, B) \mapsto \operatorname{tr}(A B)
$$


identifies $B(\mathcal{H})$ with the dual space $B_{1}(\mathcal{H})^{\prime}$ of $B_{1}(\mathcal{H})$. The set of rank one operators $P_{v, w}$ with $v, w \in \mathcal{H}^{\infty}$ is total in $B_{1}(\mathcal{H})^{\prime}$ with respect to the weak-* topology. Therefore it suffices to show that

$$
\operatorname{tr}\left((r(D) . \pi)(s) P_{v, w}\right)=\operatorname{tr}\left(\pi(s) d \pi(D) P_{v, w}\right)
$$

for all $v, w \in \mathcal{H}^{\infty}$. For $X \in \mathfrak{h}$ we have

$$
\begin{aligned}
\operatorname{tr}\left((r(X) . \pi)(s) P_{v, w}\right) & =\left.\frac{d}{d t}\right|_{t=0} \operatorname{tr}\left(\pi(s \exp t X) P_{v, w}\right) \\
& =\left.\frac{d}{d t}\right|_{t=0}\langle\pi(s) \pi(\exp t X) \cdot v, w\rangle, \\
& =\langle\pi(s) d \pi(X) \cdot v, w\rangle \\
& =\operatorname{tr}\left(\pi(s) d \pi(X) P_{v, w}\right)
\end{aligned}
$$

and therefore $(r(X) \cdot \pi)(s)=\pi(s) d \pi(X)$.

Since $\operatorname{tr}\left(\pi(s) d \pi(X) P_{v, w}\right)=\operatorname{tr}\left(\pi(s) P_{d \pi(X) \cdot v, w}\right)$, we can apply induction and obtain (ii) for general elements $D \in \mathcal{U}\left(\mathfrak{h}_{\mathbb{C}}\right)$.

(iii) is a trivial consequence of (ii) and $d \pi(D)=\chi_{\lambda}(D) 1$ for all $D \in \mathcal{Z}$.

Proposition 6.6. $\mathcal{H}_{\Theta_{\pi_{\lambda}}} \subseteq \operatorname{Hol}\left(S_{C}\right)_{\chi_{\lambda}}$.

Proof. That $\Theta=\Theta_{\pi_{\lambda}} \in \operatorname{Hol}\left(S_{C}\right)_{\chi_{\lambda}}$ follows immediately from Lemma 6.5. Now the invariance of $\operatorname{Hol}\left(S_{C}\right)_{\chi_{\lambda}}$ under $\widetilde{H} \times \widetilde{H}$ implies that $\mathcal{H}_{\Theta}^{0} \subseteq \operatorname{Hol}\left(S_{C}\right)_{\chi_{\lambda}}$ (cf. Section $2)$. Let $f \in \mathcal{H}_{\Theta}$. Then there exists a sequence of functions $f_{n} \in \mathcal{H}_{\Theta}^{0}$ such that $f_{n} \rightarrow f$ holds in the topology of uniform convergence on compact subsets of $S_{C}$ (cf. [Ne99, Prop. I.1.9]). This implies that for $D \in \mathcal{U}\left(\mathfrak{h}_{\mathbb{C}}\right)$ we have

$$
r(D) \cdot f=\lim _{n \rightarrow \infty} r(D) \cdot f_{n}
$$

and in particular

$$
r(D) \cdot f=\lim _{n \rightarrow \infty} r(D) \cdot f_{n}=\chi_{\lambda}(D) \lim _{n \rightarrow \infty} f_{n}=\chi_{\lambda}(D) f
$$

for all $D \in Z\left(\mathcal{U}\left(\mathfrak{h}_{C}\right)\right)$.

Unfortunately, it turns out that, in contrast to the situation where $S_{C}=H_{\mathbb{C}}$ is the complexification of a compact group $H$, the spaces $\operatorname{Hol}\left(S_{C}\right)_{\chi}$ may contain several characters. In view of Theorem 6.2 , this is equivalent to the observation that the assignment $\lambda \mapsto \chi_{\lambda}$ is not injective if considered as a function on a subset of the set $\widehat{S}_{C}^{\text {holo }}$ of irreducible holomorphic representations of $S_{C}$.

Example 6.7. We consider the example $\mathfrak{h}=\mathfrak{s l}(2, \mathbb{R})$ with its (up to sign) unique invariant cone $C \subseteq i \mathfrak{h}$. Then the center of the enveloping algebra is generated by the Casimir operator $\mathcal{C}$. Hence a central character is determined by its value on $\mathcal{C}$. With respect to a suitable parametrization, the irreducible representations of the simply connected semigroup $S_{C}$ are parametrized by their highest weight $\lambda \in[0, \infty[$, where $\lambda>1$ corresponds to the relative holomorphic discrete series of $\operatorname{Sl}(2, \mathbb{R})^{\sim}$, $\lambda=1$ to the limits of the holomorphic discrete series, a representation of $\mathrm{Sl}(2, \mathbb{R})$, and $\lambda=\frac{1}{2}, \frac{3}{2}$ to the irreducible constituents of the metaplectic representation. We have

$$
\chi_{\lambda}(\mathcal{C})=\frac{1}{4} \lambda(\lambda-2)
$$


Hence the representations with $\lambda \geq 1$ can be parametrized by their central characters. For $\lambda \leq 2$ we have

$$
\chi_{\lambda}=\chi_{2-\lambda} .
$$

We see in particular that $\chi_{\frac{1}{2}}=\chi_{\frac{3}{2}}$, so that the metaplectic representation has a central character.

If $\mathcal{F}$ is the Fock space of the metaplectic representation, then $\mathcal{F}=\mathcal{F}_{+} \oplus \mathcal{F}_{-}$is the decomposition into odd and even part (the irreducible representations of $S_{C}$ ). If $\Theta$ is the character of the metaplectic representation of $S_{C}$, then

$$
\mathcal{H}_{\Theta} \cong B_{2}\left(\mathcal{F}_{+}\right) \oplus B_{2}\left(\mathcal{F}_{-}\right) \subseteq \operatorname{Hol}\left(S_{C}\right)_{\chi_{\frac{1}{2}}} .
$$

Thus the holomorphic spherical functions $\Theta_{\frac{1}{2}}$ and $\Theta_{\frac{3}{2}}$ belong to the same eigenvalues of the Casimir operator $\mathcal{C}$ acting on $\operatorname{Hol}\left(S_{C}\right)$.

\section{Remarks on Possible Generalizations}

In this section we comment on the possibilities to generalize our results to more general positive domains of the type $P_{C}$ as described in Example 1.2.

The crucial point which made the arguments in Section 5 go through was the fact that for $\mathfrak{g}=\mathfrak{h}_{\mathbb{C}}$ the continuous representation theory can be reduced to the holomorphic representations theory using the Lüscher-Mack correspondence. In Section 6 the very formulation depended on the complex structure of $S_{C}$.

One possible approach to the general situation is to try to use a combination of Lüscher-Mack and the Holomorphic Extension Theorem (cf. Appendix B) to extend representations of $S_{C}$ to holomorphic representations of a suitable bigger object. The results of [KrNe96] suggest that one should restrict one's attention to symmetric Lie groups $(G, \tau)$ with a hyperbolic cone $C$ in $\mathfrak{q}$ (cf. Section 4). So, starting with a positive definite spherical function $\bar{\varphi}$ on $P_{C}$, one considers the resulting irreducible continuous representation $\left(\pi_{\varphi}, \mathcal{H}_{\varphi}\right)$ of $S_{C}$ and uses LüscherMack to find an irreducible unitary representation $(\pi, \mathcal{H})$ of $G^{c}$ related to $\pi_{\varphi}$. Let $W_{\pi} \in \mathfrak{g}^{c}$ be the cone associated to $\pi$ (cf. Definition 3.5) and set $W=\overline{W_{\pi} \cap-\tau\left(W_{\pi}\right)}$. This is a closed $G^{c}$ and $(-\tau)$-invariant convex cone in $\mathfrak{g}^{c}$ which in case it is pointed gives rise to a semigroup (cf. Appendix B)

$$
S_{W}:=\Gamma\left(\mathfrak{g}^{c}, \operatorname{algint} W\right)=G^{c} \operatorname{Exp}(i \operatorname{algint} W) \cong G^{c} \times \operatorname{algint} W,
$$

and we can extend $\pi$ to a representation of this semigroup (using the Holomorphic Extension Theorem from Appendix B), which then is again irreducible. If $W$ is also generating, then $\Gamma\left(\mathfrak{g}^{c}\right.$, algint $\left.W\right)$ simply is the $\mathrm{Ol}^{\prime}$ shanskil semigroup associated to $W$ and the extension of $\pi$ is holomorphic. Let $\sigma$ be the complex conjugation of $\mathfrak{g}_{\mathbb{C}}$ w.r.t. $\mathfrak{g}^{c}$. Then $\bar{\tau}:=\tau \circ \sigma$, where we have extended $\tau$ to a complex linear involution of $\mathfrak{g}_{\mathbb{C}}$, extends to an antiholomorphic involution of $S_{W}$ which we still denote by $\bar{\tau}$. Moreover the antiholomorphic anti-involution $g \mapsto \sigma(g)^{-1}$ on $G_{\mathbb{C}}$ gives rise to an antiholomorphic anti-involution $s \mapsto s^{*}$ on $S_{W}$ commuting with $\tau$. Thus $\left(S_{W},{ }^{*}, \bar{\tau}\right)$ is a symmetric involutive semigroup, and we can consider its positive domain $P_{W}=\left\{\bar{\tau}\left(s^{*}\right) s: s \in S_{W}\right\}$. Note that in the case that $W$ is generating, so that $S_{W}$ is an Ol'shanskiu semigroup, the results of Section 5 classify the positive definite spherical functions of the positive domain associated to $\left(S_{W},{ }^{*}\right.$, id), whereas the results of Section 6 classify the exponentially bounded holomorphic positive 
definite spherical functions on $S_{W}$. Note further that for $C_{\pi}=i W \cap \mathfrak{q}=\{X \in$ $i W: \bar{\tau}(X)=X\}$ the semigroup

$$
S_{C_{\pi}}=\left\{s \in S_{W}: \bar{\tau}(s)=s\right\} \cong H^{c} \times \operatorname{algint} C_{\pi}
$$

bears a strong similarity to $S_{C}$. In fact, if $C$ is contained in $W$ or at least intersects its algebraic interior, one can for all practical purposes replace $S_{C}$ by a suitable quotient semigroup (cf. Rem. 2.4) so that $S_{C}$ and $S_{C_{\pi}}$ intersect in a subsemigroup which is open in both, $S_{C}$ and $S_{C_{\pi}}$. The above considerations lead to the following problems:

Problem 7.1. (a) Extend the results of Section 5 to the case where $(\mathfrak{h}, \tau)$ is symmetric and $\left(S_{C},{ }^{*}\right.$, id $)$ is replaced by $\left(S_{C},{ }^{*}, \bar{\tau}\right)$, where $\bar{\tau}$ on the Lie algebra level is the complex antilinear extension of $\tau$ to $\mathfrak{h}_{\mathbb{C}}$.

(b) Given related representations $\rho$ and $\pi$ of $S_{C}$ and $G^{c}$ (in the general case of Example 1.2), find criteria under which the associated cone of $\pi$ is generating (pointed, $(-\tau)$-invariant, closed, etc.) and determine its intersection with $C$.

(c) To which extent is the spherical function $\bar{\varphi}$ of $P_{C}$ determined by the spherical functions on $P_{W}$ and $S_{W}$ obtained from the two-step extension of $\pi_{\varphi}$ via LüscherMack and the Holomorphic Extension Theorem?

(d) Determine the structure of the positive domains $P_{W}$ described above (cf. [HÓØ91], where similar constructions were used in special cases in order to construct certain Hardy spaces). For a description of this space as a bundle $G^{c} \times{ }_{H^{c}} C_{\pi}$, we refer to [KNÓ96].

\section{Appendix A: Ideals in Admissible Lie Algebras}

A Lie algebra $\mathfrak{g}$ is called admissible if $\mathfrak{g} \oplus \mathbb{R}$ contains a pointed generating invariant cone. Then $\mathfrak{g}$ contains a compactly embedded Cartan subalgebra $\mathfrak{t} \subseteq \mathfrak{g}$, and there exists a $\mathfrak{k}$-adapted positive system of roots $\Delta^{+}$(cf. [Ne99, Th. VII.3.10]). For $\alpha \in \Delta$ we put $[\alpha]:=\{\alpha,-\alpha\}$ and set $\mathfrak{g}^{[\alpha]}:=\mathfrak{g} \cap\left(\mathfrak{g}_{\mathbb{C}}^{\alpha}+\mathfrak{g}_{\mathbb{C}}^{-\alpha}\right)$.

We say that $\mathfrak{g}$ has cone potential if $\left[X_{\alpha}, \overline{X_{\alpha}}\right] \neq 0$ holds for all non-zero elements $X_{\alpha} \in \mathfrak{g}_{\mathbb{C}}^{\alpha}$. We can write $\mathfrak{g}=\mathfrak{r} \rtimes \mathfrak{s}=\mathfrak{u} \rtimes \mathfrak{l}$, where $\mathfrak{r}$ is the radical, $\mathfrak{s}$ is a $\mathfrak{t}$-invariant Levi algebra, $\mathfrak{u}$ is the nilradical, and $\mathfrak{l}$ is a reductive $\mathfrak{t}$-invariant subalgebra with $[\mathfrak{l}, \mathfrak{l}]=\mathfrak{s}$ ([HiNe93, Prop. 7.3]). For details concerning the structure theory of admissible Lie algebras we refer to [HiNe95] and [KrNe96].

We recall from [Ne94a] that a symplectic $\mathfrak{g}$-module is a symplectic vector space $(V, \Omega)$ on which $\mathfrak{g}$ acts such that the symplectic form $\Omega$ is invariant, i.e.,

$$
\Omega(X . v, w)+\Omega(v, X . w)=0 .
$$

If $\mathfrak{g}$ is reductive, then a symplectic $\mathfrak{g}$-module $(V, \Omega)$ is said to be of convex type if, first, the closed convex cone $C_{V}$ generated by the image of the moment map

$$
\Phi: V \rightarrow \mathfrak{g}^{*}, \quad\left(X \mapsto \frac{1}{2} \Omega(X . v, v)\right)
$$

is pointed, i.e., contains no vector subspace, and second, the center $\mathfrak{z}(\mathfrak{g})$ acts semisimply on $V$ with purely imaginary eigenvalues.

Lemma A.1. Let $\mathfrak{g}$ be a Lie algebra with compactly embedded Cartan subalgebra $\mathfrak{t}$ and $\mathfrak{a} \subseteq \mathfrak{g}$ an ideal intersecting int $\operatorname{comp}(\mathfrak{g})$. Then the following assertions hold:

(i) $\mathfrak{g} \subseteq \mathfrak{a}+\mathfrak{k}$ and, in particular, $\mathfrak{g}^{[\alpha]} \subseteq \mathfrak{a}$ for all $\alpha \in \Delta_{p}$. 
(ii) $\mathfrak{t}_{\mathfrak{a}}:=\mathfrak{a} \cap \mathfrak{t}$ is a compactly embedded Cartan subalgebra of $\mathfrak{a}$ and $\mathfrak{k}_{\mathfrak{a}}:=\mathfrak{k} \cap \mathfrak{a}$ is maximal compactly embedded in $\mathfrak{a}$.

(iii) $\mathfrak{g}$ has cone potential if $\mathfrak{a}$ has cone potential.

(iv) If $\mathfrak{a}$ is admissible, then $\mathfrak{g}$ is admissible.

Proof. Let $Y \in \mathfrak{a} \cap \operatorname{int} \operatorname{comp}(\mathfrak{g})$. In view of Corollary A.2.29 in [HHL89], $Y$ is contained in a compactly embedded Cartan subalgebra $\mathfrak{t}^{\prime}$ of $\mathfrak{g}$. Since compactly embeddded Cartan subalgebras are conjugate under inner automorphisms ([HHL89, Thm. A.2.13]), there exists an inner automorphism $\gamma$ with $\gamma\left(\mathfrak{t}^{\prime}\right)=\mathfrak{t}$. Hence $\gamma(Y) \in$ $\gamma(\mathfrak{a}) \cap \gamma\left(\mathfrak{t}^{\prime}\right)=\mathfrak{a} \cap \mathfrak{t}$. Therefore $X:=\gamma(Y) \in \mathfrak{t} \cap \operatorname{int} \operatorname{comp}(\mathfrak{g}) \cap \mathfrak{a}$.

(i) We consider the direct vector space decomposition $\mathfrak{g}=\mathfrak{g}_{X}+[X, \mathfrak{g}]$. Then $\mathfrak{g}_{X} \subseteq \mathfrak{k}$ ([HHL89, Thm. A.2.25]) and $[X, \mathfrak{g}] \subseteq \mathfrak{a}$ imply $\mathfrak{g} \subseteq \mathfrak{a}+\mathfrak{k}$. Since both subalgebras are invariant under $\mathfrak{t}$, we conclude hat

$$
\mathfrak{g}^{[\alpha]}=\mathfrak{a}^{[\alpha]}+\mathfrak{k}^{[\alpha]},
$$

where $\mathfrak{a}^{[\alpha]}=\mathfrak{g}^{[\alpha]} \cap \mathfrak{a}$ and $\mathfrak{k}^{[\alpha]}=\mathfrak{g}^{[\alpha]} \cap \mathfrak{k}$. From $\mathfrak{k}^{[\alpha]}=\{0\}$ for $\alpha \in \Delta_{p}$ (cf. [Ne99, Lemma VII.2.3], we get $\mathfrak{g}^{[\alpha]} \subseteq \mathfrak{a}$ for all non-compact roots $\alpha$.

(ii) It is clear that $\mathfrak{t}_{\mathfrak{a}}$ is a compactly embedded subalgebra of $\mathfrak{a}$. Let $\mathfrak{b}:=\mathfrak{z}_{\mathfrak{a}}\left(\mathfrak{t}_{\mathfrak{a}}\right)$. Then $\mathfrak{b}$ is a $\mathfrak{t}$-invariant subalgebra, hence can be written as

$$
\mathfrak{b}=\mathfrak{t}_{\mathfrak{a}}+\sum_{\alpha \in \Delta} \mathfrak{b}^{[\alpha]}
$$

From $X \in \mathfrak{t}_{\mathfrak{a}}$ and $\mathfrak{b} \subseteq \mathfrak{g}_{X} \subseteq \mathfrak{k}$ we deduce that $\mathfrak{b}^{[\alpha]}=\{0\}$ for $\alpha \in \Delta_{p}$.

Suppose that $\mathfrak{b}^{[\alpha]} \neq\{0\}$. Then $\alpha$ is a compact root and $\mathfrak{g}^{[\alpha]}=\mathfrak{b}^{[\alpha]}$ follows from $\operatorname{dim}_{\mathbb{C}} \mathfrak{g}_{\mathbb{C}}^{\alpha}=1$ (cf. [Ne99, Lemma VII.2.3]). Let $\mathfrak{s}$ be a $\mathfrak{t}$-invariant Levi complement of $\mathfrak{g}$. Then $\mathfrak{g}^{[\alpha]} \subseteq[\mathfrak{k}, \mathfrak{k}] \subseteq \mathfrak{s}$. Moreover $\mathfrak{s}_{\mathfrak{a}}:=\mathfrak{s} \cap \mathfrak{a}$ is an ideal of $\mathfrak{s}$ intersecting $\mathfrak{g}^{[\alpha]}$, so that $\mathfrak{g}^{[\alpha]} \subseteq \mathfrak{s}_{\mathfrak{a}}$ follows. On the other hand $\mathfrak{t} \cap \mathfrak{s}$ is a compactly embedded Cartan subalgebra of $\mathfrak{s}$, which implies that $\mathfrak{t} \cap \mathfrak{s}_{\mathfrak{a}}$ is a compactly embedded Cartan subalgebra of $\mathfrak{s}_{\mathfrak{a}}$. Thus $\mathfrak{z}_{\mathfrak{s}_{\mathfrak{a}}}\left(\mathfrak{t} \cap \mathfrak{s}_{\mathfrak{a}}\right)=\mathfrak{t} \cap \mathfrak{s}_{\mathfrak{a}} \subseteq \mathfrak{t}_{\mathfrak{a}}$, which gives $\mathfrak{g}^{[\alpha]} \subseteq \mathfrak{z}_{\mathfrak{s}_{\mathfrak{a}}}\left(\mathfrak{t} \cap \mathfrak{s}_{\mathfrak{a}}\right)$, and therefore a contradiction. This proves that $\mathfrak{b}=\mathfrak{t}_{\mathfrak{a}}$, hence that $\mathfrak{t}_{\mathfrak{a}}$ is a Cartan subalgebra of $\mathfrak{a}$.

It is clear that $\mathfrak{k}_{\mathfrak{a}}$ is a compactly embedded subalgebra of $\mathfrak{a}$. Suppose that $\widetilde{\mathfrak{k}}_{\mathfrak{a}} \supseteq \mathfrak{k}_{\mathfrak{a}}$ is maximal compactly embedded in $\mathfrak{a}$. Then $\left[\widetilde{\mathfrak{k}}_{\mathfrak{a}}, \widetilde{\mathfrak{k}}_{\mathfrak{a}}\right]$ is a semisimple compact subalgebra of $\mathfrak{a}$, hence compactly embedded in $\mathfrak{g}$. Moreover $\mathfrak{t}_{\mathfrak{a}}$ is a Cartan subalgebra of $\widetilde{\mathfrak{k}}_{\mathfrak{a}}$, so that $\left.\mathfrak{t}_{\mathfrak{a}}=\mathfrak{z}\left(\widetilde{\mathfrak{k}}_{\mathfrak{a}}\right) \oplus\left(\widetilde{\mathfrak{k}}_{\mathfrak{a}}, \widetilde{\mathfrak{k}}_{\mathfrak{a}}\right] \cap \mathfrak{t}_{\mathfrak{a}}\right)$. Hence $\mathfrak{t}_{\mathfrak{a}}+\left[\widetilde{\mathfrak{k}}_{\mathfrak{a}}, \widetilde{\mathfrak{k}}_{\mathfrak{a}}\right]$ is a compactly embedded subalgebra of $\mathfrak{g}$. The uniqueness of $\widetilde{\mathfrak{k}}_{\mathfrak{a}}$ shows that it is invariant under $\mathfrak{t}$, and hence $\mathfrak{t}+\left[\widetilde{\mathfrak{k}}_{\mathfrak{a}}, \widetilde{\mathfrak{k}}_{\mathfrak{a}}\right]$ is a subalgebra of $\mathfrak{g}$ which is then automatically compactly embedded, i.e. contained in $\mathfrak{k}$. Thus

$$
\widetilde{\mathfrak{k}}_{\mathfrak{a}}=\mathfrak{z}\left(\widetilde{\mathfrak{k}}_{\mathfrak{a}}\right)+\left[\widetilde{\mathfrak{k}}_{\mathfrak{a}}, \widetilde{\mathfrak{k}}_{\mathfrak{a}}\right] \subseteq \mathfrak{t}_{\mathfrak{a}}+\mathfrak{k}_{\mathfrak{a}}=\mathfrak{k}_{\mathfrak{a}}
$$

proves that $\mathfrak{k}_{\mathfrak{a}}$ is maximal compactly embedded in $\mathfrak{a}$.

(iii) Let $\alpha \in \Delta_{p}$ be a non-compact root and $X_{\alpha} \in \mathfrak{g}_{\mathbb{C}}^{\alpha} \backslash\{0\}$. If $\beta:=\left.\alpha\right|_{\mathfrak{t}_{\mathfrak{a}}}$, then $X_{\alpha} \in \mathfrak{a}_{\mathbb{C}}^{\beta}$, and since $\alpha\left(\left[X_{\alpha}, \overline{X_{\alpha}}\right]\right) \geq 0$, the root $\beta$ of $\mathfrak{a}_{\mathbb{C}}$ is non-compact. Therefore the fact that $\mathfrak{a}$ has cone potential implies that $\left[X_{\alpha}, \overline{X_{\alpha}}\right] \neq 0$. Thus $\mathfrak{g}$ has cone potential.

(iv) Suppose that $\mathfrak{a}$ is an admissible Lie algebra. Then (iii) implies that the Lie algebra $\mathfrak{g}$ has cone potential. Therefore the nilradical $\mathfrak{u}$ of $\mathfrak{g}$ is a Heisenberg algebra with $\mathfrak{u}=[\mathfrak{t}, \mathfrak{u}] \oplus \mathfrak{z}(\mathfrak{g}),[\mathfrak{u}, \mathfrak{u}] \subseteq \mathfrak{z}(\mathfrak{g})$, and there exists a reductive subalgebra $\mathfrak{l} \subseteq \mathfrak{g}$ 
such that $\mathfrak{t}_{\mathfrak{l}}:=\mathfrak{t} \cap \mathfrak{l}$ is a compactly embedded Cartan subalgebra of $\mathfrak{l}, \mathfrak{t}=\mathfrak{z}(\mathfrak{g}) \oplus \mathfrak{t}_{\mathfrak{l}}$, and $\mathfrak{g}=\mathfrak{u} \rtimes \mathfrak{l}([$ HiNe93, Thm. 7.15]).

If $\mathfrak{z}(\mathfrak{g})=\{0\}$, then the fact that $\mathfrak{g}$ has cone potential implies that $\mathfrak{u}=\{0\}$ and hence that $\mathfrak{g}$ is reductive. Then the fact that $\mathfrak{a}$ contains all non-compact simple ideals of $\mathfrak{g}$ (cf. (i)) entails that the commutator algebra of $\mathfrak{g}$ contains only compact and hermitian simple ideals. Hence $\mathfrak{g}$ is quasihermitian and therefore admissible (cf. [HiNe93, Thm. 7.26]).

Now we assume that $\mathfrak{z}(\mathfrak{g}) \neq\{0\}$. We consider the vector space $V:=[\mathfrak{t}, \mathfrak{u}]$ as a module of the reductive Lie algebra $\mathfrak{l}_{\mathfrak{a}}:=\mathfrak{l} \cap \mathfrak{a}$. According to [Ne94a, Prop. V.6], there exists a functional $\beta \in \mathfrak{z}^{*}$ such that the symplectic form $\Omega$ on $V$ defined by $\Omega(v, w)=\beta([v, w])$ for $v, w \in V$ turns $(V, \Omega)$ into a symplectic $\mathfrak{l}_{\mathfrak{a}}$-module of convex type. Let $\Psi: V \rightarrow \mathfrak{I}^{*}$ denote the moment map given by $\Psi(v)(Y)=\frac{1}{2} \Omega(Y . v, v)$. Since $\mathfrak{a} \cap \mathfrak{t}$ contains the element $X$, we see that $V_{\text {fix, } \mathfrak{l}_{\mathfrak{a}}}:=\left\{v \in V: \mathfrak{l}_{\mathfrak{a}} \cdot v=\{0\}\right\}=\{0\}$ because this space is contained in the centralizer of $X$. Moreover $\mathfrak{z}\left(\mathfrak{l}_{\mathfrak{a}}\right) \subseteq \mathfrak{t}$ acts semisimply with purely imaginary spectrum. We recall from (ii) above that the subalgebra $\mathfrak{k}_{\mathfrak{a}}:=\mathfrak{k} \cap \mathfrak{a} \subseteq \mathfrak{a}$ is maximal compactly embedded. Hence [Ne94a, Prop.

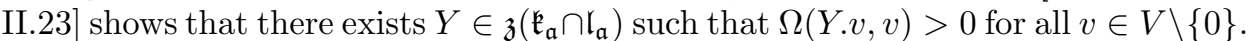

We write $\mathfrak{l}=\mathfrak{l}_{\mathfrak{a}} \oplus \mathfrak{l}_{1}$, where $\mathfrak{l}_{1}$ is a complementary ideal for $\mathfrak{l}_{\mathfrak{a}}$ in $\mathfrak{l}$. Then $\mathfrak{l}_{1}$ is a compact Lie algebra commuting with $\mathfrak{l}_{\mathfrak{a}}$. Moreover $\mathfrak{k} \cap \mathfrak{l}=\mathfrak{l}_{1} \oplus\left(\mathfrak{k}_{\mathfrak{a}} \cap \mathfrak{l}_{\mathfrak{a}}\right)$. Therefore $\mathfrak{z}\left(\mathfrak{k}_{\mathfrak{a}} \cap \mathfrak{l}_{\mathfrak{a}}\right) \subseteq \mathfrak{z}(\mathfrak{k} \cap \mathfrak{l})$, and we can apply [Ne94a, Prop. II.23] again to see that $(V, \Omega)$ is also of convex type as a symplectic module of the reductive Lie algebra l. Finally Theorem V.1 in [Ne94a] now shows that the Lie algebra $\mathfrak{g}$ contains pointed generating invariant cones, hence it is in particular admissible.

Lemma A.2. (a) If $W \subseteq \mathfrak{g}$ is a pointed closed convex invariant cone, then Spec $(\operatorname{ad} X) \subseteq i \mathbb{R}$ holds for all $X \in W$.

(b) If $C \subseteq \mathfrak{g}^{*}$ is a closed convex generating invariant subset, then

$$
\operatorname{Spec}(\operatorname{ad} X) \subseteq i \mathbb{R}
$$

for all $X \in \overline{B(C)}$, where

$$
B(C):=\{Y \in \mathfrak{g}: \sup \langle Y, C\rangle<\infty\} .
$$

Proof. (a) Let $\mathfrak{a}:=W-W$. Then $\mathfrak{a}$ is an ideal in $\mathfrak{g}$. For $X \in W$ we have $\operatorname{ad} X(\mathfrak{g}) \subseteq \mathfrak{a}$, hence $\operatorname{Spec}(\operatorname{ad} X) \subseteq \operatorname{Spec}\left(\left.\operatorname{ad} X\right|_{\mathfrak{a}}\right) \cup\{0\}$. Thus we may w.l.o.g. assume that $W$ is generating.

Moreover, since the spectrum, as a set, depends continuously on ad $X$, it suffices to prove the assertion for $X \in \operatorname{int} W$. This follows immediately from the fact that int $W \subseteq \operatorname{int} \operatorname{comp}(\mathfrak{g})$ ([HHL89, Thm. III.2.14]).

(b) Let $C \subseteq \mathfrak{g}^{*}$ be a closed convex generating invariant subset, and put $W:=$ cone $(C \times\{1\}) \subseteq \mathfrak{g}^{*} \times \mathbb{R}$. An element $X \in \mathfrak{g}$ is contained in $B(C)$ if and only if there exists $\lambda>0$ with $(-X, \lambda) \in W^{\star}$. So it suffices to show that $\operatorname{Spec}(\operatorname{ad} Y) \subseteq i \mathbb{R}$ holds for all $Y \in W^{\star} \subseteq \mathfrak{g} \times \mathbb{R}$.

Since $C$ is generating, there are two possibilities. If $C$ contains interior points, then the same holds for $W$ and so $W^{\star}$ is pointed. Hence the assertion follows from (a). If $C$ has no interior points, then it is contained in a proper affine hyperplane $H$. Let $Y \in \mathfrak{g}$ denote the uniquely determined element with $\langle Y, H\rangle=\{1\}$. Then $W^{\star}=\mathbb{R}(Y,-1) \oplus\left(W^{\star} \cap \mathfrak{g}\right)$. Since the hyperplane $H$ containing $C$ is invariant under the coadjoint action, the element $Y \in \mathfrak{g}$ is invariant under the adjoint action, hence 
$Y \in \mathfrak{z}(\mathfrak{g})$. From that and the fact that $W^{\star} \cap \mathfrak{g}$ is pointed, we conclude with (a) that for each element $Z \in W^{\star}$ we have $\operatorname{Spec}(\operatorname{ad} Z) \subseteq i \mathbb{R}$.

\section{Appendix B: The Holomorphic Extension Theorem}

In this appendix we prove an important generalization of a result of $\mathrm{Ol}^{\prime}$ shanskiu ([HiNe93, Prop. 9.18], cf. also [Ols82]) which says that a unitary representation $\pi$ of $G$ with discrete kernel, for which the (closed) cone

$$
W_{\pi, 0}=\{X \in \mathfrak{g}: i d \pi(X) \leq 0\}
$$

is generating, can be extended to a contraction representation of $G \exp \left(i W_{\pi, 0}\right)$.

Let $G$ be a simply connected Lie group and $(\pi, \mathcal{H})$ be a continuous unitary representation of $G$ with discrete kernel. Consider the associated cone

$$
W_{\pi}:=\left\{X \in \mathfrak{g}: \sup \left\langle X, I_{\pi}\right\rangle<\infty\right\}
$$

of $\pi$, where $I_{\pi}$ is the moment set for $\pi$ (cf. Definition 3.5). Then [Ne99, Prop. X.1.5] says that for $X \in \mathfrak{g}$ we have

$$
\sup \operatorname{Spec}(i d \pi(X))=\sup \left\langle X, I_{\pi}\right\rangle
$$

(cf. (3.2)). We denote the algebraic interior of $W_{\pi}$, i.e., the interior w.r.t. the linear span of $W_{\pi}$, by algint $W_{\pi}$. The cone $W_{\pi}$ is not necessarily closed, but we have

$$
\operatorname{algint} W_{\pi}=\operatorname{algint} \overline{W_{\pi}} .
$$

Lemma A.2(b) implies that the cone $W:=\overline{W_{\pi}}=\overline{B\left(I_{\pi}\right)}$ satisfies

$$
\operatorname{Spec}(\operatorname{ad} X) \subseteq \mathbb{R}
$$

for all $X \in i W$, i.e., the cone $i W \subseteq i \mathfrak{g}$ is regular. Now Lawson's Theorem ([HiNe93, Cor. 7.35]) implies that the semigroup $S_{V}$ generated by the exponential image of $V=\mathfrak{g}+i W$ in the simply connected group $G_{\mathbb{C}}$ with Lie algebra $\mathfrak{g}_{\mathbb{C}}$ is given by

$$
S_{V}=G_{1} \exp (i W) \cong G_{1} \times W,
$$

where $G_{1}$ is the analytic subgroup of $G_{\mathbb{C}}$ with Lie algebra $\mathfrak{g}$. The simply connected covering semigroup of $S_{V}$ will be denoted $\Gamma(\mathfrak{g}, W)$. Similarly the simply connected covering of $G_{1} \exp (i$ algint $W)$ will be denoted $\Gamma(\mathfrak{g}$, algint $W)$. The lift of the exponential map to $\Gamma(\mathfrak{g}$, algint $W$ ) will be written Exp. Then the decomposition (B.2) lifts to the $O l^{\prime}$ shanskiu decomposition

$$
\Gamma(\mathfrak{g}, W)=G \operatorname{Exp}(i W) \cong G \times W .
$$

Note that $W-W$ is an ideal in $\mathfrak{g}$, so that the subgroup $\widetilde{H}_{W}$ of $G$ generated by $\exp (W-W)$ is closed and simply connected. Similarly the subgroup $\left(H_{W}\right)_{\mathbb{C}}$ of $G_{\mathbb{C}}$ generated by the exponential image of $(W-W)_{\mathbb{C}}$ is closed and simply connected. Moreover the analytic subgroup $H_{W}$ of $\left(H_{W}\right)_{\mathbb{C}}$ with Lie algebra $W-W$ is closed. Then $\left(H_{W}\right)_{\mathbb{C}}$ contains an $\mathrm{Ol}^{\prime}$ shanskiı̌ semigroup $\left(H_{W}\right) \exp (i W) \cong H_{W} \times W$, and the corresponding simply connected covering $\Gamma(W-W, W)$ is homeomorphic to $\widetilde{H}_{W} \times W$. Again we denote the simply connected covering of $H_{W} \exp (i \operatorname{algint} W)$ by $\Gamma(W-W$, algint $W)$ and obtain an Ol' shanskiu decomposition

$$
\Gamma(W-W, W)=\widetilde{H}_{W} \operatorname{Exp}(i W) \cong \widetilde{H}_{W} \times W .
$$


Theorem B (The Holomorphic Extension Theorem). Let $G$ be a simply connected Lie group and $(\pi, \mathcal{H})$ a continuous unitary representation with discrete kernel. Then there exists a strongly continuous involutive representation

$$
\widehat{\pi}: \Gamma\left(\mathfrak{g}, \operatorname{algint} W_{\pi}\right) \cong G \times \operatorname{Exp}\left(i \operatorname{algint} W_{\pi}\right) \rightarrow B(\mathcal{H})
$$

such that

(1) $\widehat{\pi}(g s)=\pi(g) \widehat{\pi}(s)$ for all $g \in G$ and $s \in \Gamma\left(\mathfrak{g}\right.$, algint $\left.W_{\pi}\right)$, and

(2) $\widehat{\pi}$ is holomorphic on the open complex Ol'shanskiน semigroup

$$
\Gamma\left(W_{\pi}-W_{\pi}, \text { algint } W_{\pi}\right) .
$$

Proof. Step 1: We define the mapping $\widehat{\pi}$ via the $\mathrm{Ol}^{\prime}$ shanskil decomposition of $\Gamma\left(\mathfrak{g}, W_{\pi}\right)$ by

$$
\widehat{\pi}(g \operatorname{Exp}(X))=\pi(g) e^{d \pi(X)}
$$

and note that $X \in i W_{\pi}$ implies that the operator $e^{d \pi(X)}$ is bounded with

$$
\log \|\widehat{\pi}(\operatorname{Exp} X)\|=\sup \operatorname{Spec}(d \pi(X))=\sup \left\langle-i X, I_{\pi}\right\rangle .
$$

From

$$
\pi(g) e^{d \pi(X)} \pi(g)^{-1}=e^{\pi(g) d \pi(X) \pi(g)^{-1}}=e^{d \pi(\operatorname{Ad}(g) X)},
$$

we see that

$$
\begin{aligned}
\widehat{\pi}\left((g \operatorname{Exp} X)^{*}\right) & =\widehat{\pi}\left(\operatorname{Exp} X g^{-1}\right)=\widehat{\pi}\left(g^{-1} \operatorname{Exp} \operatorname{Ad}(g) X\right) \\
& =\pi(g)^{-1} e^{d \pi(\operatorname{Ad}(g) X)}=e^{d \pi(X)} \pi(g)^{-1}=\widehat{\pi}(g \operatorname{Exp} X)^{*},
\end{aligned}
$$

i.e., $\widehat{\pi}\left(s^{*}\right)=\widehat{\pi}(s)^{*}$.

Step 2: Local boundedness on $G \operatorname{Exp}\left(\operatorname{algint} W_{\pi}\right)$ : According to (B.5), we have

$$
\log \|\widehat{\pi}(g \operatorname{Exp} X)\|=\log \|\widehat{\pi}(\operatorname{Exp} X)\|=\sup \left\langle-i X, I_{\pi}\right\rangle,
$$

which defines a function on $G \times$ algint $W_{\pi}$ which is independent of the first argument and convex on the cone algint $W_{\pi}$. Hence it is continuous and therefore locally bounded.

Step 3: Using [HiNe93, Lemma 9.16], we get a 0-neighborhood $U_{\mathbb{C}} \subseteq \mathfrak{g}_{\mathbb{C}}$ and a dense subspace $\mathcal{H}_{1} \subseteq \mathcal{H}$ such that the mappings

$$
\gamma_{v}: U_{\mathbb{C}} \rightarrow \mathcal{H}, \quad X \mapsto e^{d \pi(X)} . v, \quad v \in \mathcal{H}_{1},
$$

are holomorphic, and $\gamma_{v}(X)=\pi(\exp X) . v$ holds for all $X \in \mathfrak{g} \cap U_{\mathbb{C}}$. We conclude that there exists an open convex $e$-neighborhood $B$ in $\mathfrak{g}$ such that $V:=$ $\operatorname{Exp}(B) \operatorname{Exp}\left(i\left(B \cap \operatorname{algint} W_{\pi}\right)\right)$ is an open $e$-neighborhood in $\Gamma\left(\mathfrak{g}\right.$, algint $\left.W_{\pi}\right)$, and there exists a set of continuous mappings $\alpha_{v}: V \rightarrow \mathcal{H}$ satisfying

$$
\alpha_{v}(g)=\pi(g) \cdot v \quad \text { for } \quad g \in \operatorname{Exp}(B)=G \cap V
$$

and which are holomorphic on $V^{0}:=V \cap \Gamma\left(W-W\right.$, algint $\left.W_{\pi}\right)$.

Step 4: Strong continuity on $\Gamma\left(\mathfrak{g}\right.$, algint $\left.W_{\pi}\right)$ : We know in particular that the mappings

$$
\gamma_{v}^{\prime}: \operatorname{Exp}\left(U_{\mathbb{C}} \cap i W_{\pi}\right) \rightarrow \mathcal{H}, \quad \operatorname{Exp}(X) \mapsto \widehat{\pi}(\operatorname{Exp} X) \cdot v=e^{d \pi(X)} \cdot v
$$

are continuous for $v \in \mathcal{H}_{1}$. Let $s_{0} \in \Gamma\left(\mathfrak{g}\right.$, algint $\left.W_{\pi}\right) \cap \operatorname{Exp}\left(U_{\mathbb{C}} \cap i\right.$ algint $\left.W_{\pi}\right)$ and use Step 3 to find a neighborhood $N$ of $s$ in $\operatorname{Exp}\left(U_{\mathbb{C}} \cap i\right.$ algint $\left.W_{\pi}\right)$ and $m \in \mathbb{R}$ such that $\|\widehat{\pi}(s)\| \leq m$ for all $s \in N$. 
If $v_{n} \in \mathcal{H}_{1}$ with $v_{n} \rightarrow v \in \mathcal{H}$, then the choice of $N$ implies that $\gamma_{v_{n}}^{\prime}$ converges uniformly to $\gamma_{v}^{\prime}$ on $N$. Since $\mathcal{H}_{1}$ is dense in $\mathcal{H}$, we conclude that $\widehat{\pi}$ is strongly continuous on $\operatorname{Exp}\left(U_{\mathbb{C}} \cap i\right.$ algint $\left.W_{\pi}\right)$.

Let $v \in \mathcal{H}, X \in i$ algint $W_{\pi}$, and $X_{n} \in i$ algint $W_{\pi}$ with $X_{n} \rightarrow X$. We choose $m \in \mathbb{N}$ such that $\frac{1}{m} X_{n} \in U_{\mathbb{C}} \cap i$ algint $W_{\pi}$ for all $n \in \mathbb{N}$. Then, in view of [HiNe93, Prop. 9.2(ii)] and Step 1, we see that

$$
\widehat{\pi}\left(\operatorname{Exp}\left(X_{n}\right)\right) \cdot v=\widehat{\pi}\left(\operatorname{Exp}\left(\frac{1}{m} X_{n}\right)\right)^{m} \cdot v \rightarrow \widehat{\pi}\left(\operatorname{Exp}\left(\frac{1}{m} X\right)\right)^{m} \cdot v=\widehat{\pi}(\operatorname{Exp} X) \cdot v .
$$

Thus $\widehat{\pi}$ is strongly continuous on $\operatorname{Exp}\left(i\right.$ algint $\left.W_{\pi}\right)$. Since it is also strongly continuous on G, [HiNe93, Prop. 9.2] and Lawson's Theorem ([HiNe93, Thm. 7.34]) imply that $\widehat{\pi}$ is strongly continuous on $\Gamma\left(\mathfrak{g}\right.$, algint $\left.W_{\pi}\right)$.

Step 5: $\widehat{\pi}$ is holomorphic on $V^{0}$ : Let $v \in \mathcal{H}_{1}$. We claim that $\widehat{\pi}(s) \cdot v=\alpha_{v}(s)$ for all $s \in V$. Fix $s=g \operatorname{Exp}(X) \in V$. Then the construction of $V$ shows that $g \operatorname{Exp}([0,1] X) \subseteq V$. For $z \in \mathbb{C}$ with $\operatorname{Re} z \geq 0$ we set $\gamma(z):=g \operatorname{Exp}(z X)$. Let $U:=\gamma^{-1}(V)$. Then

$$
z \mapsto \widehat{\pi}(\gamma(z)) \cdot v \quad \text { and } \quad \alpha_{v} \circ \gamma
$$

are holomorphic on $\gamma^{-1}\left(V^{0}\right)$ ([HiNe93, Prop. 9.9]), continuous on $U$, and equal on $U \cap i \mathbb{R}$. Now [HiNe93, Lemma 9.12(ii)] entails that these mappings are equal on the connected component of 0 in $U$, and we obtain in particular that

$$
\alpha_{v}(g \operatorname{Exp} X)=\widehat{\pi}(g \operatorname{Exp} X) \cdot v .
$$

If $v_{n} \rightarrow v, v_{n} \in \mathcal{H}_{1}$ and $v \in \mathcal{H}$, then the same argument as in Step 4 and $\widehat{\pi}(s) \cdot v_{n}=\alpha_{v_{n}}(s)$ imply that $\alpha_{v_{n}}$ converges locally uniformly to $\alpha_{v}$ with $\widehat{\pi}(s) \cdot v=$ $\alpha_{v}(s)$ for $s \in V^{0}$. Since $\mathcal{H}_{1}$ is dense in $\mathcal{H}$, we conclude that $\widehat{\pi}$ is strongly holomorphic on $V^{0}$. Finally, [HiNe93, Lemma 9.7(i)] implies that $\widehat{\pi}$ is a holomorphic operator valued mapping on $V^{0}$.

Step 6: Analyticity on $\operatorname{Exp}\left(i\right.$ algint $\left.W_{\pi}\right)$ : Let $X \in i$ algint $W_{\pi}$, and let $W \subseteq$ $i$ algint $W_{\pi}$ be a compact neighborhood of $X$. Then there exists $m \in \mathbb{N}$ such that $\operatorname{Exp}\left(\frac{1}{m} W\right) \subseteq V^{0}$. Since the $m$-times multiplication mapping $B(\mathcal{H})^{m} \rightarrow B(\mathcal{H})$ is real analytic, we conclude that the mapping

$$
W \rightarrow B(\mathcal{H}), \quad Y \mapsto e^{d \pi(Y)}=\left(e^{\frac{1}{m} d \pi(Y)}\right)^{m}
$$

is real analytic.

Step 7: $\widehat{\pi}$ is holomorphic on $\Gamma\left(W-W\right.$, algint $\left.W_{\pi}\right)$ : The analyticity of $s \mapsto$ $\langle\widehat{\pi}(s) . v, w\rangle$ with $w \in \mathcal{H}^{\omega}$ follows from the Ol'shanskiì decomposition and Step 6 . Consequently this mapping is also holomorphic on $\Gamma\left(W-W\right.$, algint $\left.W_{\pi}\right)$, because, in view of the analyticity, the domain where the Cauchy-Riemann equations are satisfied is extendable via analytic continuation. Now [HiNe93, Lemma 9.7(i)] implies operator valued holomorphy.

Step 8: $\widehat{\pi}$ is a homomorphism: From the definition of $\widehat{\pi}$ it is clear that $\widehat{\pi}(g s)=$ $\pi(g) \widehat{\pi}(s)$ holds for all $s \in \Gamma\left(\mathfrak{g}\right.$, algint $\left.W_{\pi}\right)$ and $g \in G$. Similarly, the fact that $\widehat{\pi}$ respects the involution implies that $\widehat{\pi}(s g)=\widehat{\pi}(s) \pi(g)$.

Let $s=\exp (Y) g \in \Gamma\left(\mathfrak{g}\right.$, algint $\left.W_{\pi}\right)$, where $g \in G$ and $Y \in i$ algint $W_{\pi}$, and $X \in W_{\pi}$. Then the mapping

$$
\gamma_{X}: \mathbb{R}+i \mathbb{R}^{+} \rightarrow B(\mathcal{H}), \quad z \mapsto e^{z d \pi(X)},
$$


is strongly continuous and holomorphic on the upper half plane ([Ne95a, Thm. III.1]). Let $v, w \in \mathcal{H}$ and $s \in \Gamma$ (g, algint $\left.W_{\pi}\right)$, and put

$$
f_{1}(z):=\langle\widehat{\pi}(\operatorname{Exp}(z X) s) \cdot v, w\rangle=\langle\widehat{\pi}(\operatorname{Exp}(z X) \operatorname{Exp}(Y)) \pi(g) \cdot v, w\rangle
$$

and

$$
f_{2}(z):=\left\langle\gamma_{X}(z) \widehat{\pi}(s) \cdot v, w\right\rangle .
$$

Then the fact that $\left.\widehat{\pi}\right|_{\Gamma\left(W-W \text {,algint } W_{\pi}\right)}$ is holomorphic implies that $f_{1}$ is holomorphic on int $\mathbb{C}^{+}$and continuous on $\mathbb{C}^{+}$. Since the function $f_{2}$ has the same properties and, for $x \in \mathbb{R}$,

$$
f_{1}(x)=\langle\widehat{\pi}(\operatorname{Exp}(x X) s) \cdot v, w\rangle=\langle\widehat{\pi}(\operatorname{Exp}(x X)) \widehat{\pi}(s) \cdot v, w\rangle=f_{2}(x),
$$

we conclude with [HiNe93, Lemma 9.17] that $f_{1}=f_{2}$. Since $v, w \in \mathcal{H}$ are arbitrary, it follows in particular that

$$
\widehat{\pi}(\operatorname{Exp}(i X) s)=\widehat{\pi}(\operatorname{Exp}(i X)) \widehat{\pi}(s)
$$

for all $s \in \Gamma\left(\mathfrak{g}\right.$, algint $\left.W_{\pi}\right)$ and $X \in W_{\pi}$. Thus

$$
\widehat{\pi}\left(s_{1} s_{2}\right)=\widehat{\pi}\left(s_{1}\right) \widehat{\pi}\left(s_{2}\right)
$$

holds for $s_{1} \in \Gamma\left(\mathfrak{g}\right.$, algint $\left.W_{\pi}\right)$ and $s_{2} \in \Gamma\left(\mathfrak{g}, \operatorname{algint} W_{\pi}\right)$.

Let $X \in \operatorname{algint} i W_{\pi}$. Then $\operatorname{Exp}(X) \in \Gamma\left(\mathfrak{g}\right.$, algint $\left.W_{\pi}\right)$, and the operator

$$
\widehat{\pi}(\operatorname{Exp} X)=e^{d \pi(X)}
$$

has a dense range. For $v=\widehat{\pi}(\operatorname{Exp} X) \cdot w, w \in \mathcal{H}$, we obtain for

$$
s_{1}, s_{2} \in \Gamma\left(\mathfrak{g}, \operatorname{algint} W_{\pi}\right)
$$

that

$$
\begin{aligned}
\widehat{\pi}\left(s_{1} s_{2}\right) \cdot v & =\widehat{\pi}\left(s_{1} s_{2}\right) \widehat{\pi}(\operatorname{Exp} X) \cdot w=\widehat{\pi}\left(s_{1} s_{2} \operatorname{Exp} X\right) \cdot w \\
& =\widehat{\pi}\left(s_{1}\right) \widehat{\pi}\left(s_{2} \operatorname{Exp} X\right) \cdot w=\widehat{\pi}\left(s_{1}\right) \widehat{\pi}\left(s_{2}\right) \widehat{\pi}(\operatorname{Exp} X) \cdot w=\widehat{\pi}\left(s_{1}\right) \widehat{\pi}\left(s_{2}\right) \cdot v
\end{aligned}
$$

But the operators $\widehat{\pi}\left(s_{1} s_{2}\right)$ and $\widehat{\pi}\left(s_{1}\right) \widehat{\pi}\left(s_{2}\right)$ are both bounded; hence they coincide on the whole space $\mathcal{H}$.

\section{REFERENCES}

[Ar50] N. Aronszajn, Theory of reproducing kernels, Transactions of the Amer. Math. Soc. 68 (1950), 337-404. MR 14:479c

[FHÓ94] J. Faraut, J. Hilgert, and G. Ólafsson, Spherical functions on ordered symmetric spaces, Ann. Inst. Fourier 44 (1994), 927-966. MR 96a:43012

[Hel84] S. Helgason, Groups and geometric analysis, Academic Press, Orlando (1984). MR 86c: 22017

[HHL89] J. Hilgert, K. H. Hofmann, and J. D. Lawson, Lie Groups, Convex Cones, and Semigroups, Oxford University Press (1989). MR 91k:22020

[HiNe93] J. Hilgert and K. - H. Neeb, Lie semigroups and their applications, Springer Lecture Notes in Math. 1552 (1993). MR 96j:22002

[HiNe95] J. Hilgert and K. - H. Neeb, Compression semigroups of open orbits on complex manifolds, Arkiv för Mat. 33 (1995), 293-322. MR 97j:22013

[HiNe96] J. Hilgert and K. - H. Neeb, Spherical functions on Ol'shanskiน spaces, J. Funct. Anal. 142 (1996), 446-493. MR 98c:43015

[HÓ96] J. Hilgert and G. Ólafsson, G., Causal Symmetric Spaces, Geometry and Harmonic Analysis, Academic Press (1996). MR 97m:43006

[HÓØ91] J. Hilgert, G. Ólafsson, and B. Ørsted, Hardy Spaces on Affine Symmetric Spaces, J. reine angew. Math. 415 (1991), 189-218. MR 92h:22030

[KrNe96] B. Krötz and K.-H. Neeb, On Hyperbolic Cones and Mixed Symmetric Spaces, Journal of Lie Theory 6:1 (1996), 69-146. MR 97k:17007 
[KNÓ96] B. Krötz, K.-H. Neeb, and G. Ólafsson, Spherical Representations and Mixed Symmetric Spaces, Representations Theory (1997), 424-461. (electronic) MR 99a:22031.

[Lo69] O. Loos, Symmetric Spaces I : General Theory, Benjamin, New York, Amsterdam (1969). MR 39:365a

[LM75] M. Lüscher and G. Mack, Global Conformal Invariance in Quantum Field Theory, Comm. Math. Phys. 41 (1975), 203-234. MR 51:7503

[Ne94a] K.-H. Neeb, The classification of Lie algebras with invariant cones, Journal of Lie Theory 4:2 (1994), 1-46. MR 96e:17013

[Ne94b] K.-H. Neeb, Holomorphic representation theory II, Acta Math. 173:1 (1994), 103-133. MR 96a:22025

[Ne94c] K.-H. Neeb, A convexity theorem for semisimple symmetric spaces, Pac. J. Math. 162:2 (1994), 305-349. MR 95b:22016

[Ne95a] K.-H. Neeb, Holomorphic representation theory I, Math. Ann. 301 (1995), 155-181. MR 96a:22024

[Ne95b] K.-H. Neeb, Square integrable highest weight representations, Glasgow Math. J. 39 (1987), 295-321. MR 99a:22021

[Ne96] K.-H. Neeb, Coherent states, holomorphic extensions, and highest weight representations, Pac. J. Math. 174:2 (1996), 497-542. MR 97k:22018

[Ne99] K.-H. Neeb, Holomorphy and Convexity in Lie Theory, Expositions in Mathematics, de Gruyter, to appear.

[Nel70] E. Nelson, Analytic vectors, Ann. of Math. 70 (1959), 572-615. MR 21:5901

[Ols82] G. I. Ol'shanskiū, Invariant cones in Lie algebras, Lie semigroups, and the holomorphic discrete series, Funct. Anal. and Appl. 15 (1982), 275-285. MR 83e:32032

[RS72] M. Reed and B. Simon, Methods of Mathematical Physics I: Functional Analysis, Academic Press, New York (1972). MR 58:12429a

[Wa72] G. Warner, Harmonic analysis on semi-simple Lie groups I, Springer, Berlin (1972). MR 58:16979

Mathematisches Institut, Technische Universität Clausthal, Erzstr. 1, 38678 Clausthal-Zellerfeld, Germany

E-mail address: hilgert@math.tu-clausthal.de

Mathematisches Institut, Universität Erlangen, Bismarckstr. $1 \frac{1}{2}, 91054$ Erlangen, Germany

E-mail address: neeb@mi.uni.erlangen.de

Current address: Fachbereich Mathematik, Technische Universität Darmstadt, Schloßgartenstr. 7, 64289 Darmstadt, Germany 